\title{
Mechanical Properties and Structural Performance of Recycled Aggregate Concrete: An Overview
}

\author{
Mahdi Arezoumandi ${ }^{1 *}$, Ehsan Ghafari ${ }^{2}$, Seyedhamed Sadati ${ }^{3}$ and Mostafa Fakharifar ${ }^{3}$ \\ ${ }^{1}$ Department of Civil, Architectural and Environmental Engineering, Shahab Danesh University, Iran \\ ${ }^{2}$ Purdue University, USA \\ ${ }^{3}$ Department of Civil, Architectural and Environmental Engineering, Missouri University of Science and Technology, USA
}

Submission: August 02, 2017; Published: September 14, 2017

*Corresponding author: Mahdi Arezoumandi, Department of Civil, Architectural and Environmental Engineering, Shahab Danesh University, Iran, Tel: 0015732024703; Email: ma526@mst.edu

\begin{abstract}
Using Recycled Concrete Aggregate (RCA) in concrete has been the subject of many studies for years. A comprehensive literature review on mechanical properties of Recycled Aggregate Concrete (RAC) including splitting tensile strength, modulus of elasticity, flexural strength, and fracture mechanic is presented. Furthermore, this paper summarizes previous studies on structural behavior (shear, flexural, and bond) of RAC. In addition, databases are created for both mechanical properties and structural performances of RAC in order to lead to changes or acceptance in design codes and standards' provisions. Additionally, mechanical properties and structural strengths of RAC were compared with both the U.S. and European standards. Results of this study show that existing code provisions are not conservative for both mechanical properties and structural strengths of RAC.
\end{abstract}

Keywords: Mechanical Properties; Structural performance; Recycled Concrete Aggregate

\section{Introduction}

Around 25 billion tons of concrete is used each year globally that means over 3.8 tons per person in the world each year [1]. Occupying about $60 \%$ to $70 \%$ of the volume, aggregate is a main ingredient of concrete. Nowadays, there is an increasing trend toward using sustainable concrete and reusing of aggregates from demolished concrete is one way to achieve this aim. Although using recycled concrete aggregate (RCA) does not lead to significant reduction of $\mathrm{CO}_{2}$ emission, but it reduces using natural resources (virgin aggregate) as well as helping to reduce dumping construction and demolition wastes in landfills. More than 900 million tons of construction and demolition waste is produced each year only in Europe, the U.S., and Japan [1]. The use of RCA to make a new concrete began in Europe after World War II. Also the building contractors' society of Japan started research on RCA after the 1973 oil crisis [2]. Although comprehensive research has been done on fresh and hardened properties of recycled aggregate concrete (RAC), relatively limited studies have been performed on structural behavior of RAC. This paper compiles around 60 published studies (from 1977 to 2015) that studied mechanical properties (splitting tensile strength, modulus of elasticity, flexural strength, and fracture mechanic) as well as structural performance (shear, flexural, and bond) of RAC. The work presented here summarized only those studies that used RCA as a coarse aggregate replacement. Given the fact that incorporation of fine RCA is typically reported to result in inferior fresh and hardened properties, the present study summarizes the properties of RCA incorporating coarse RCA [3].

\section{Mechanical Properties}

The following section compares splitting tensile strength and flexural strength as well as modulus of elasticity and fracture energy of RAC with the ACI 318, CEB-FIP, Eurocode 2 and JSCE provisions $[4,7]$.

\section{Splitting tensile strength}

Table $1(\mathrm{a}, \mathrm{c})$ summarizes the compressive strength $\left(f_{c}^{\prime}\right)$ splitting tensile strength $\left(f_{c t}\right)$ and ratio of the experimental-tocode values for splitting tensile strength based on the ACI 318 [5] and Eurocode 2 [7] provisions for previous studies [8-28]. The average values of experimental-to-code ratio are 0.90 and 0.92 for the ACI 318 [5] and Eurocode 2 [7] provisions, respectively. Both provisions are conservative for about $22 \%$ of the data points. In other words, the investigated codes overestimate the splitting tensile strength for RAC (Figure 1) based on measured compressive strength values. Furthermore, Regression analysis was conducted to draw the best fit and $95 \%$ confidence intervals for the investigated data points. The lower and upper 95\% 
confidence intervals (L95 and U95) are established. Results of the statistical data analysis reveal the fact that even in the case of the mixtures made with $100 \%$ RCA replacement (RAC-100), test results fall within a $95 \%$ confidence interval of a nonlinear regression curve fit of the conventional concrete (CC) database (Figure 1).

Table 1 (a-c): Summary of splitting tensile strength test results.

\begin{tabular}{|c|c|c|c|c|c|c|c|c|c|c|c|c|c|}
\hline Author & Year & $\begin{array}{l}\text { RCA } \\
(\%)\end{array}$ & $\mathrm{f}^{\prime} \mathrm{c}$ & fct & $\begin{array}{l}\text { Test/ } \\
\text { ACI }\end{array}$ & $\begin{array}{l}\text { Test/ } \\
\text { EC2 }\end{array}$ & Author & Year & RCA & $\mathrm{f}_{c}^{\prime}$ & $\mathrm{f}_{\mathrm{ct}}$ & $\begin{array}{l}\text { Test/ } \\
\text { ACI }\end{array}$ & $\begin{array}{l}\text { Test/ } \\
\text { EC2 }\end{array}$ \\
\hline \multirow{3}{*}{ Sagoe et al. [8] } & \multirow{3}{*}{2001} & \multirow{3}{*}{100} & 27 & 3.2 & 1.1 & 1.18 & \multirow{14}{*}{$\begin{array}{c}\text { Ajdukiewicz } \\
\& \\
\text { Kliszczewicz } \\
\text { (9) }\end{array}$} & \multirow{14}{*}{2002} & \multirow{14}{*}{100} & 52.1 & 3.8 & 0.94 & 0.9 \\
\hline & & & 31 & 3.7 & 1.19 & 1.25 & & & & 24.3 & 2 & 0.72 & 0.77 \\
\hline & & & 29 & 2.7 & 0.9 & 0.95 & & & & 35.9 & 2.9 & 0.86 & 0.91 \\
\hline \multirow{27}{*}{$\begin{array}{l}\text { Ajdukiewicz \& } \\
\text { Kliszczewicz [9] }\end{array}$} & \multirow{27}{*}{2002} & \multirow{27}{*}{100} & 18.2 & 1.9 & 0.8 & 0.95 & & & & 41.8 & 3.3 & 0.91 & 0.92 \\
\hline & & & 29.7 & 2.9 & 0.95 & 1 & & & & 46 & 3.6 & 0.95 & 0.95 \\
\hline & & & 37.1 & 3.3 & 0.97 & 0.98 & & & & 49.6 & 3.8 & 0.96 & 0.93 \\
\hline & & & 41.3 & 3.7 & 1.03 & 1.04 & & & & 53.8 & 4.1 & 1 & 0.98 \\
\hline & & & 44.5 & 4 & 1.07 & 1.05 & & & & 55.4 & 4.3 & 1.03 & 1.02 \\
\hline & & & 47.1 & 4.3 & 1.12 & 1.1 & & & & 13 & 1.4 & 0.69 & 0.82 \\
\hline & & & 49.3 & 4.5 & 1.14 & 1.1 & & & & 31.7 & 2.3 & 0.73 & 0.77 \\
\hline & & & 23.9 & 2.3 & 0.84 & 0.92 & & & & 42.3 & 2.9 & 0.8 & 0.81 \\
\hline & & & 35.4 & 2.7 & 0.81 & 0.84 & & & & 48.2 & 3.6 & 0.93 & 0.9 \\
\hline & & & 39.3 & 3 & 0.85 & 0.86 & & & & 54.4 & 4 & 0.97 & 0.95 \\
\hline & & & 42.3 & 3.2 & 0.88 & 0.89 & & & & 58.7 & 4.4 & 1.03 & 1 \\
\hline & & & 46.1 & 3.4 & 0.89 & 0.87 & \multirow{6}{*}{$\begin{array}{l}\text { Etxeberria et } \\
\text { al. (10) }\end{array}$} & \multirow{6}{*}{2007} & \multirow{2}{*}{25} & 38.8 & 3.01 & 0.86 & 0.89 \\
\hline & & & 49.6 & 3.6 & 0.91 & 0.88 & & & & 46.3 & 3.88 & 1.02 & 0.99 \\
\hline & & & 53.1 & 3.8 & 0.93 & 0.9 & & & \multirow{2}{*}{50} & 39.4 & 3.36 & 0.96 & 0.96 \\
\hline & & & 24.6 & 2.3 & 0.83 & 0.82 & & & & 44.4 & 3.65 & 0.98 & 0.96 \\
\hline & & & 38.2 & 3 & 0.87 & 0.88 & & & \multirow{2}{*}{100} & 38.3 & 2.79 & 0.81 & 0.82 \\
\hline & & & 44.3 & 3.4 & 0.91 & 0.89 & & & & 38.7 & 3.28 & 0.94 & 0.96 \\
\hline & & & 48.5 & 3.7 & 0.95 & 0.93 & \multirow{9}{*}{$\begin{array}{c}\text { Ajdukiewicz } \\
\text { \& } \\
\text { Kliszczewicz } \\
\text { (11) }\end{array}$} & \multirow{9}{*}{2007} & \multirow{9}{*}{100} & 34.6 & 2.6 & 0.79 & 0.81 \\
\hline & & & 52.5 & 4 & 0.99 & 0.95 & & & & 56.4 & 3.3 & 0.78 & 0.79 \\
\hline & & & 55.4 & 4.2 & 1.01 & 1 & & & & 40.1 & 2.9 & 0.82 & 0.83 \\
\hline & & & 56.1 & 4.3 & 1.03 & 1.02 & & & & 60.2 & 4.3 & 0.99 & 0.98 \\
\hline & & & 14.2 & 1.8 & 0.85 & 1 & & & & 85.3 & 5.3 & 1.02 & 1.08 \\
\hline & & & 30.4 & 2.7 & 0.87 & 0.93 & & & & 35.3 & 3 & 0.9 & 0.94 \\
\hline & & & 36.8 & 3 & 0.88 & 0.91 & & & & 57.6 & 3.7 & 0.87 & 0.86 \\
\hline & & & 41.3 & 3.3 & 0.92 & 0.92 & & & & 36.6 & 2.9 & 0.86 & 0.88 \\
\hline & & & 45.2 & 3.5 & 0.93 & 0.92 & & & & 35.8 & 3.2 & 0.96 & 0.97 \\
\hline & & & 48.8 & 3.7 & 0.95 & 0.93 & & & & & & & \\
\hline
\end{tabular}




\section{Civil Engineering Research Journal}

\begin{tabular}{|c|c|c|c|c|c|c|c|c|c|c|c|c|c|}
\hline Author & Year & $\begin{array}{l}\text { RCA } \\
\text { (\%) }\end{array}$ & $f^{\prime} c$ & fct & $\begin{array}{l}\text { Test/ } \\
\text { ACI }\end{array}$ & $\begin{array}{l}\text { Test/ } \\
\text { EC2 }\end{array}$ & Author & Year & $\begin{array}{l}\text { RCA } \\
\text { (\%) }\end{array}$ & $f^{\prime} c$ & fct & $\begin{array}{l}\text { Test/ } \\
\text { ACI }\end{array}$ & $\begin{array}{l}\text { Test/ } \\
\text { EC2 }\end{array}$ \\
\hline \multirow{4}{*}{$\begin{array}{c}\text { Sato et al. } \\
\text { [12] }\end{array}$} & \multirow{4}{*}{2007} & \multirow{4}{*}{100} & 30.4 & 2.6 & 0.84 & 0.9 & \multirow{6}{*}{$\begin{array}{c}\text { Kou et al. } \\
\text { [18] }\end{array}$} & \multirow{6}{*}{2013} & \multirow{3}{*}{50} & 34.3 & 2.21 & 0.67 & 0.69 \\
\hline & & & 28.4 & 2.4 & 0.8 & 0.86 & & & & 48.8 & 3.12 & 0.8 & 0.76 \\
\hline & & & 34.5 & 2.8 & 0.85 & 0.88 & & & & 55.4 & 3.64 & 0.87 & 0.87 \\
\hline & & & 31.8 & 3.3 & 1.04 & 1.1 & & & \multirow{3}{*}{100} & 35.6 & 2.26 & 0.68 & 0.71 \\
\hline \multirow{4}{*}{$\begin{array}{c}\text { Belén \& } \\
\text { Fernando [3] }\end{array}$} & \multirow{4}{*}{2007} & \multirow{4}{*}{50} & 39.7 & 3.4 & 0.96 & 0.97 & & & & 49.2 & 3.07 & 0.78 & 0.75 \\
\hline & & & 39.2 & 3.3 & 0.94 & 0.94 & & & & 54.1 & 3.52 & 0.85 & 0.84 \\
\hline & & & 41.5 & 3.4 & 0.94 & 0.94 & \multirow{24}{*}{$\begin{array}{l}\text { Abd Elhakam } \\
\text { et al. [19] }\end{array}$} & \multirow{24}{*}{2012} & \multirow{12}{*}{25} & 21 & 1.65 & 0.64 & 0.75 \\
\hline & & & 40.5 & 3.4 & 0.95 & 0.97 & & & & 26.7 & 2.18 & 0.75 & 0.81 \\
\hline \multirow{6}{*}{$\begin{array}{c}\text { Yang et al. } \\
\text { [13] }\end{array}$} & \multirow{6}{*}{2008} & \multirow{2}{*}{30} & 36.7 & 4.03 & 1.19 & 1.22 & & & & 28.1 & 2.35 & 0.79 & 0.84 \\
\hline & & & 32.6 & 3.21 & 1 & 1.07 & & & & 32.2 & 2.98 & 0.94 & 0.96 \\
\hline & & \multirow{4}{*}{100} & 38 & 3.65 & 1.06 & 1.07 & & & & 38.3 & 3.24 & 0.93 & 0.95 \\
\hline & & & 30.4 & 2.85 & 0.92 & 0.98 & & & & 41.7 & 3.83 & 1.06 & 1.06 \\
\hline & & & 36 & 3.49 & 1.04 & 1.09 & & & & 20.2 & 1.97 & 0.78 & 0.9 \\
\hline & & & 29.5 & 2.56 & 0.84 & 0.88 & & & & 27.4 & 2.31 & 0.79 & 0.83 \\
\hline \multirow{8}{*}{$\begin{array}{c}\text { Tabsh \& } \\
\text { Abdelfatah } \\
\text { [14] }\end{array}$} & \multirow{8}{*}{2009} & \multirow{8}{*}{100} & 36 & 4 & 1.19 & 1.25 & & & & 31.1 & 2.88 & 0.92 & 0.96 \\
\hline & & & 25 & 2.9 & 1.04 & 1.12 & & & & 32.3 & 2.65 & 0.83 & 0.85 \\
\hline & & & 23 & 2.8 & 1.04 & 1.17 & & & & 38.6 & 2.94 & 0.85 & 0.86 \\
\hline & & & 36 & 4 & 1.19 & 1.25 & & & & 41.5 & 3.83 & 1.06 & 1.06 \\
\hline & & & 52 & 4.7 & 1.16 & 1.12 & & & \multirow{12}{*}{75} & 20 & 0.98 & 0.39 & 0.45 \\
\hline & & & 50 & 4.8 & 1.21 & 1.17 & & & & 21.5 & 1.41 & 0.54 & 0.61 \\
\hline & & & 48 & 4.2 & 1.08 & 1.05 & & & & 22.7 & 1.78 & 0.67 & 0.74 \\
\hline & & & 47 & 4 & 1.04 & 1.03 & & & & 27.2 & 1.59 & 0.54 & 0.59 \\
\hline \multirow{2}{*}{$\begin{array}{l}\text { Fathifazl et } \\
\text { al. [15] }\end{array}$} & \multirow{2}{*}{2009} & 63.5 & 41.6 & 3.4 & 0.94 & 0.94 & & & & 34.9 & 2.53 & 0.76 & 0.79 \\
\hline & & 74.3 & 49.1 & 3.7 & 0.94 & 0.9 & & & & 35.4 & 2.82 & 0.85 & 0.88 \\
\hline & & 30 & 24.7 & 2.4 & 0.86 & 0.92 & & & & 20.7 & 1.47 & 0.58 & 0.77 \\
\hline $\begin{array}{c}\text { Choi et al. } \\
\text { [16] }\end{array}$ & 2010 & 50 & 24.2 & 2.3 & 0.83 & 0.88 & & & & 25.9 & 2.18 & 0.76 & 0.99 \\
\hline & & 100 & 22.6 & 2.3 & 0.86 & 0.96 & & & & 30.1 & 2.35 & 0.76 & 0.81 \\
\hline \multirow{4}{*}{$\begin{array}{c}\text { Butler et al. } \\
{[17]}\end{array}$} & \multirow{4}{*}{2011} & \multirow{4}{*}{100} & 30.9 & 2.8 & 0.9 & 0.93 & & & & 27.4 & 2.18 & 0.74 & 0.78 \\
\hline & & & 47.9 & 3.4 & 0.88 & 0.92 & & & & 35.7 & 2.88 & 0.86 & 0.9 \\
\hline & & & 31.3 & 2.3 & 0.73 & 0.77 & & & & 37.5 & 3.59 & 1.05 & 1.06 \\
\hline & & & 49.4 & 3.1 & 0.79 & 0.76 & & & & & & & \\
\hline
\end{tabular}




\begin{tabular}{|c|c|c|c|c|c|c|c|c|c|c|c|c|c|}
\hline Author & Year & $\begin{array}{l}\text { RCA } \\
(\%)\end{array}$ & $\mathbf{f}_{c}^{\prime}$ & $\mathbf{f}_{\mathrm{ct}}$ & $\begin{array}{l}\text { Test/ } \\
\text { ACI }\end{array}$ & $\begin{array}{l}\text { Test/ } \\
\text { EC2 }\end{array}$ & Author & Year & $\begin{array}{l}\text { RCA } \\
\text { (\%) }\end{array}$ & $\mathbf{f}_{c}^{\prime}$ & $\mathbf{f}_{\mathrm{ct}}$ & $\begin{array}{l}\text { Test/ } \\
\text { ACI }\end{array}$ & $\begin{array}{l}\text { Test/ } \\
\text { EC2 }\end{array}$ \\
\hline \multirow{2}{*}{$\begin{array}{c}\text { Ignjatovic et al. } \\
{[20]}\end{array}$} & \multirow{2}{*}{2013} & 50 & 44.2 & 2.7 & 0.73 & 0.71 & \multirow{2}{*}{$\begin{array}{c}\text { Kou \& Poon } \\
{[23]}\end{array}$} & \multirow{2}{*}{2013} & \multirow{2}{*}{100} & 62.7 & 4.83 & 1.09 & 1.1 \\
\hline & & 100 & 42.5 & 3.2 & 0.88 & 0.86 & & & & 52.2 & 4.41 & 1.09 & 1.08 \\
\hline \multirow{6}{*}{ Butler et al. [21] } & \multirow{6}{*}{2013} & \multirow{6}{*}{100} & 38.6 & 3.51 & 1.01 & 1.03 & \multirow{2}{*}{$\begin{array}{l}\text { Arezoumandi } \\
\text { et al. [24] }\end{array}$} & \multirow{2}{*}{2014} & \multirow{2}{*}{100} & 30 & 2.55 & 0.83 & 0.88 \\
\hline & & & 38.1 & 3.11 & 0.9 & 0.91 & & & & 34.1 & 2.65 & 0.81 & 0.83 \\
\hline & & & 39.3 & 3.3 & 0.94 & 0.94 & \multirow{5}{*}{$\begin{array}{c}\text { Kang et al. } \\
\text { [25] }\end{array}$} & \multirow{5}{*}{2014} & 15 & 59.4 & 3.5 & 0.81 & 0.8 \\
\hline & & & 60.1 & 3.84 & 0.88 & 0.87 & & & 30 & 48.8 & 3.4 & 0.87 & 0.85 \\
\hline & & & 60.2 & 3.7 & 0.85 & 0.84 & & & 15 & 32.7 & 3 & 0.94 & 0.97 \\
\hline & & & 62.8 & 3.72 & 0.84 & 0.85 & & & 30 & 31.7 & 2.7 & 0.86 & 0.9 \\
\hline \multirow{6}{*}{ Kim \& Yun [22] } & \multirow{6}{*}{2013} & 30 & 33.8 & 2.5 & 0.77 & 0.81 & & & 50 & 29 & 2.7 & 0.9 & 0.93 \\
\hline & & 60 & 32.4 & 2.7 & 0.85 & 0.87 & \multirow{6}{*}{$\begin{array}{c}\text { Folino \& } \\
\text { Xargay [26] }\end{array}$} & \multirow{6}{*}{2014} & 30 & 39.2 & 3.87 & 1.1 & 1.11 \\
\hline & & 100 & 29.3 & 2.7 & 0.89 & 0.93 & & & 60 & 30.4 & 3.9 & 1.26 & 1.34 \\
\hline & & 30 & 31.5 & 2.7 & 0.86 & 0.9 & & & 100 & 29.1 & 3.32 & 1.1 & 1.14 \\
\hline & & 60 & 30.7 & 2.1 & 0.68 & 0.72 & & & 100 & 36.5 & 2.5 & 0.74 & 0.76 \\
\hline & & 100 & 29.5 & 1.8 & 0.59 & 0.62 & & & 50 & 50.6 & 3.2 & 0.8 & 0.78 \\
\hline \multirow{17}{*}{ Kou \& Poon [23] } & \multirow{17}{*}{2013} & \multirow{10}{*}{50} & 42.5 & 3.16 & 0.87 & 0.85 & & & 100 & 45.1 & 4.1 & 1.09 & 1.08 \\
\hline & & & 41.3 & 3.9 & 1.08 & 1.08 & \multirow{8}{*}{$\begin{array}{c}\text { Butler et al. } \\
{[27]}\end{array}$} & \multirow{8}{*}{2014} & \multirow{8}{*}{100} & 30.7 & 2.8 & 0.9 & 0.97 \\
\hline & & & 51.2 & 3.51 & 0.88 & 0.86 & & & & 38.6 & 3.5 & 1.01 & 1.03 \\
\hline & & & 47.1 & 3.41 & 0.89 & 0.85 & & & & 48.2 & 3.4 & 0.87 & 0.85 \\
\hline & & & 55.6 & 3.92 & 0.94 & 0.93 & & & & 60.1 & 3.9 & 0.9 & 0.89 \\
\hline & & & 50.6 & 3.62 & 0.91 & 0.88 & & & & 31.1 & 2.3 & 0.74 & 0.77 \\
\hline & & & 61.4 & 4.41 & 1.01 & 1 & & & & 49.4 & 3.1 & 0.79 & 0.76 \\
\hline & & & 55.1 & 4.14 & 1 & 0.99 & & & & 39.3 & 3.3 & 0.94 & 0.94 \\
\hline & & & 65.3 & 4.71 & 1.04 & 1.07 & & & & 62.8 & 3.7 & 0.83 & 0.84 \\
\hline & & & 57.5 & 4.32 & 1.02 & 1 & \multirow{3}{*}{ Steele [28] } & 2014 & 50 & 24.5 & 2.2 & 0.79 & 0.85 \\
\hline & & \multirow{7}{*}{100} & 38.1 & 3.06 & 0.89 & 0.9 & & & 100 & 33.4 & 2.2 & 0.68 & 0.73 \\
\hline & & & 36.5 & 2.98 & 0.88 & 0.9 & & & & & & & \\
\hline & & & 46.6 & 3.56 & 0.93 & 0.91 & \multicolumn{5}{|c|}{ Ave. } & 0.9 & 0.92 \\
\hline & & & 43.1 & 3.44 & 0.94 & 0.93 & & & & & & & \\
\hline & & & 51.1 & 4.12 & 1.03 & 1 & & & & & & & \\
\hline & & & 46.2 & 3.78 & 0.99 & 0.97 & & & & & & & \\
\hline & & & 56.3 & 4.45 & 1.06 & 1.06 & & & & & & & \\
\hline
\end{tabular}



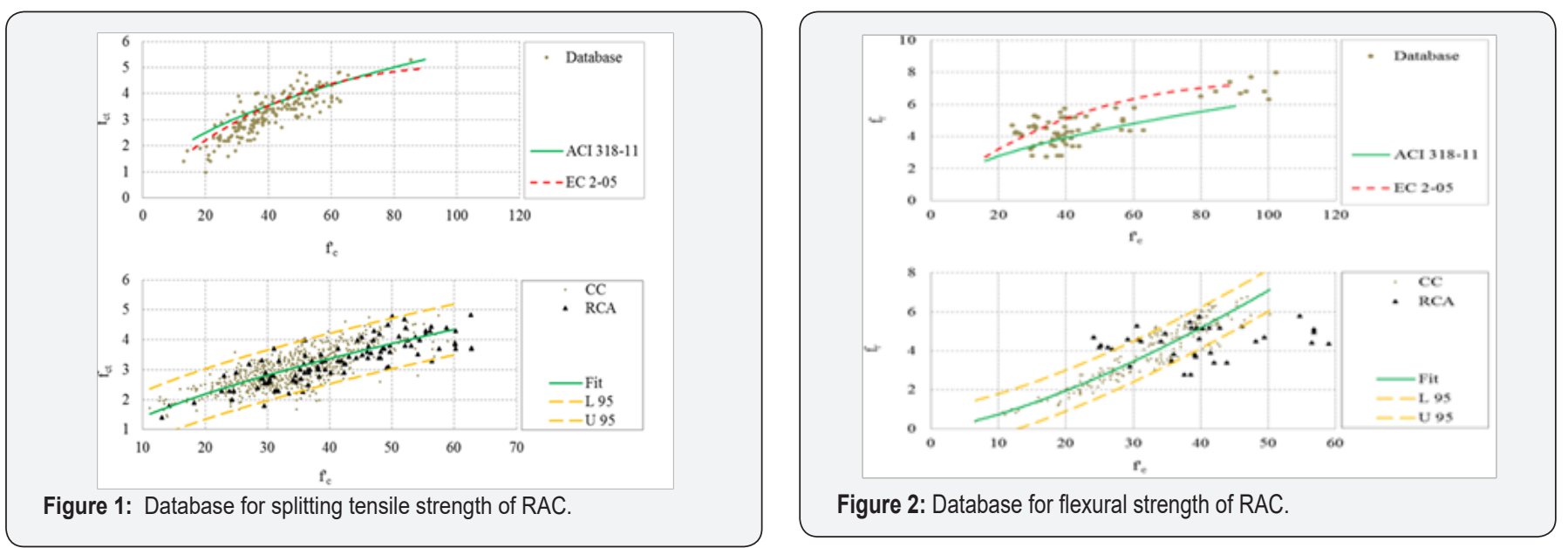

Table 2 (a): Summary of flexural strength test results.

\begin{tabular}{|c|c|c|c|c|c|c|c|c|c|c|c|c|c|}
\hline Author & Year & $\begin{array}{l}\text { RCA } \\
\text { (\%) }\end{array}$ & $\mathbf{f}^{\prime}{ }_{c}$ & $f_{r}$ & $\begin{array}{c}\text { Test/ } \\
\text { ACI }\end{array}$ & $\begin{array}{c}\text { Test/ } \\
\text { EC }\end{array}$ & Author & Year & $\begin{array}{l}\text { RCA } \\
\text { (\%) }\end{array}$ & $\mathbf{f}_{c}^{\prime}$ & $f_{r}$ & $\begin{array}{c}\text { Test/ } \\
\text { ACI }\end{array}$ & $\begin{array}{c}\text { Test/ } \\
\text { EC }\end{array}$ \\
\hline \multirow{3}{*}{ Katz [29] } & \multirow{3}{*}{2003} & \multirow{3}{*}{100} & 24.1 & 4.7 & 1.54 & 1.25 & \multirow{11}{*}{$\begin{array}{c}\text { Butler et al. } \\
\text { [21] }\end{array}$} & \multirow{11}{*}{2013} & \multirow{11}{*}{100} & 46 & 5.26 & 1.25 & 0.96 \\
\hline & & & 30.5 & 5.3 & 1.55 & 1.26 & & & & 42.8 & 5.2 & 1.28 & 0.98 \\
\hline & & & 29.1 & 4.6 & 1.38 & 1.11 & & & & 39.7 & 5.77 & 1.48 & 1.14 \\
\hline \multirow{6}{*}{$\begin{array}{c}\text { Yang et al. } \\
\text { [13] }\end{array}$} & \multirow{6}{*}{2008} & \multirow{2}{*}{30} & 36.7 & 4.21 & 1.12 & 0.88 & & & & 39.7 & 4.62 & 1.18 & 0.91 \\
\hline & & & 32.6 & 3.59 & 1.01 & 0.8 & & & & 40.3 & 5.16 & 1.31 & 1.02 \\
\hline & & \multirow{2}{*}{50} & 38 & 4.01 & 1.05 & 0.81 & & & & 41.2 & 5.18 & 1.3 & 1.01 \\
\hline & & & 30.4 & 3.4 & 0.99 & 0.81 & & & & 54.7 & 5.8 & 1.26 & 0.95 \\
\hline & & \multirow{2}{*}{100} & 36 & 3.84 & 1.03 & 0.82 & & & & 56.8 & 4.95 & 1.06 & 0.8 \\
\hline & & & 29.5 & 3.2 & 0.95 & 0.76 & & & & 56.8 & 5.11 & 1.09 & 0.83 \\
\hline \multirow{11}{*}{$\begin{array}{c}\text { Schubert et } \\
\text { al. [30] }\end{array}$} & \multirow{11}{*}{2012} & \multirow{3}{*}{100} & 34.7 & 4.9 & 1.34 & 1.06 & & & & 56.4 & 4.43 & 0.95 & 0.72 \\
\hline & & & 38.4 & 5.5 & 1.43 & 1.12 & & & & 59 & 4.36 & 0.92 & 0.68 \\
\hline & & & 34.1 & 4.5 & 1.24 & 0.97 & \multirow{9}{*}{$\begin{array}{c}\text { Andreu \& } \\
\text { Miren [31] }\end{array}$} & \multirow{9}{*}{2014} & 20 & 88.5 & 7.4 & 1.27 & 1.02 \\
\hline & & \multirow{4}{*}{50} & 31.7 & 5.2 & 1.49 & 1.2 & & & 50 & 94.8 & 7.7 & 1.28 & 1.04 \\
\hline & & & 30.7 & 5.3 & 1.54 & 1.26 & & & 100 & 93.4 & 6.8 & 1.13 & 0.92 \\
\hline & & & 30.6 & 4.7 & 1.37 & 1.12 & & & 20 & 102.1 & 8 & 1.28 & 1 \\
\hline & & & 31 & 4.6 & 1.33 & 1.08 & & & 50 & 98.8 & 6.8 & 1.1 & 0.9 \\
\hline & & \multirow{4}{*}{100} & 25.1 & 4.3 & 1.38 & 1.14 & & & 100 & 100.1 & 6.3 & 1.02 & 0.82 \\
\hline & & & 24.9 & 4.2 & 1.36 & 1.12 & & & 20 & 91.7 & 6.7 & 1.13 & 0.93 \\
\hline & & & 26.2 & 4.2 & 1.32 & 1.09 & & & 50 & 84.4 & 6.8 & 1.19 & 0.96 \\
\hline & & & 26.8 & 4.1 & 1.28 & 1.07 & & & 100 & 79.9 & 6.5 & 1.17 & 0.94 \\
\hline
\end{tabular}

Table 2 (b): Summary of flexural strength test results.

\begin{tabular}{|c|c|c|c|c|c|c|c|c|c|c|c|c|c|}
\hline Author & Year & $\begin{array}{l}\text { RCA } \\
\text { (\%) }\end{array}$ & $\mathbf{f}_{c}^{\prime}$ & $f_{r}$ & $\begin{array}{c}\text { Test/ } \\
\text { ACI }\end{array}$ & $\begin{array}{l}\text { Test/ } \\
\text { EC }\end{array}$ & Author & Year & $\begin{array}{l}\text { RCA } \\
(\%)\end{array}$ & $f^{\prime} c$ & fr & $\begin{array}{c}\text { Test/ } \\
\text { ACI }\end{array}$ & $\begin{array}{c}\text { Test/ } \\
\text { EC }\end{array}$ \\
\hline \multirow{2}{*}{$\begin{array}{l}\text { Arezoumandi } \\
\text { et al. [24] }\end{array}$} & \multirow{2}{*}{2014} & \multirow{2}{*}{100} & 30 & 2.81 & 0.82 & 0.68 & \multirow{7}{*}{$\begin{array}{c}\text { Knaack \& } \\
\text { Kurama } \\
{[32]}\end{array}$} & \multirow{7}{*}{2014} & 50 & 40 & 3.5 & 0.89 & 0.69 \\
\hline & & & 34.1 & 2.76 & 0.77 & 0.62 & & & 50 & 39.3 & 4.1 & 1.05 & 0.81 \\
\hline \multirow{5}{*}{$\begin{array}{c}\text { Butler et al. } \\
{[27]}\end{array}$} & \multirow{5}{*}{2014} & \multirow{5}{*}{100} & 30.7 & 4.6 & 1.34 & 1.1 & & & 100 & 43.8 & 3.4 & 0.83 & 0.62 \\
\hline & & & 38.6 & 5.2 & 1.35 & 1.04 & & & 100 & 38.5 & 2.8 & 0.73 & 0.57 \\
\hline & & & 48.2 & 4.5 & 1.05 & 0.78 & & & 50 & 43.6 & 4.4 & 1.07 & 0.81 \\
\hline & & & 60.1 & 5.8 & 1.21 & 0.91 & & & 50 & 40.2 & 4.3 & 1.09 & 0.85 \\
\hline & & & 31.1 & 4.5 & 1.3 & 1.07 & & & 100 & 41.4 & 3.9 & 0.98 & 0.76 \\
\hline
\end{tabular}




\section{Civil Engineering Research Journal}

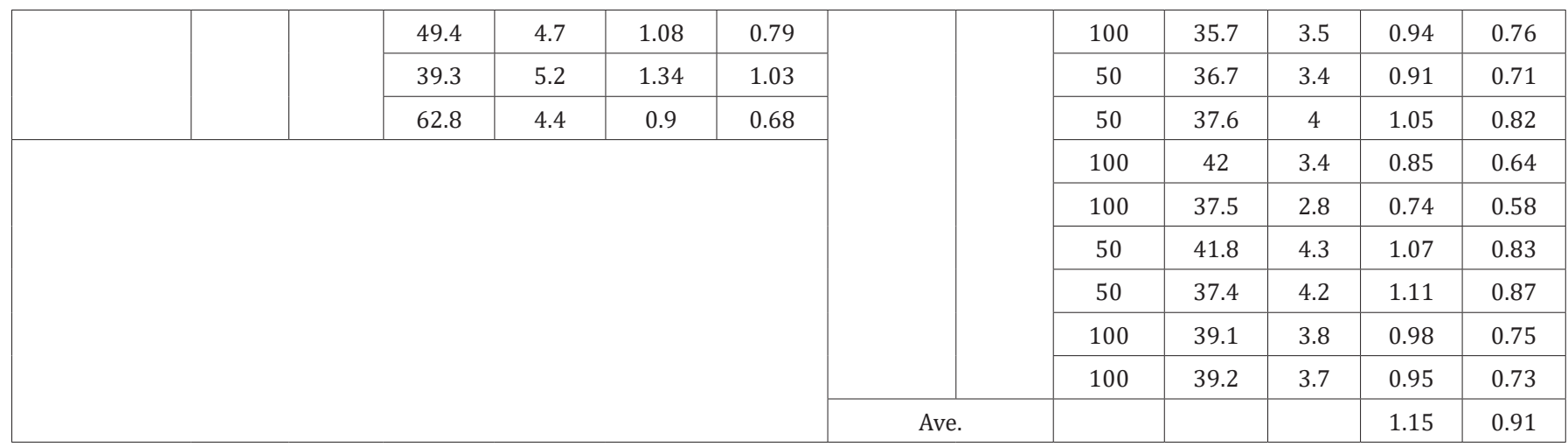

\section{Flexural strength}

Figure 2 presents the flexural strength database of RAC mixtures compared to the provisions of both ACI 318 [5] and Eurocode 2 [6]. Based on the observed trends, it may be concluded that both provisions over predict flexural strength of RAC. However, the ACI 318 [4] is unconservative for $24 \%$ of the data points compared to overestimations observed for about $65 \%$ of the data points in the case of the EC $2[6]$. Table $2(a, b)$ summarizes the compressive strength, fc, flexural strength, $\mathrm{fr}$, and the experimental-to-code ratios for flexural strength based on the ACI 318 [4] and EC 2 [6] provisions for previous studies from 2003 to 2014. The average values of the experimental-tocode ratios are 1.15 for the ACI 318 [4] and 0.92 for the EC 2 [6] provision. This reveals the fact that the ACI 318 underestimates the flexural strength of RAC by $15 \%$, proposing ACI 318 as a reliable source for predicting the flexural strength of the RCA. Contrary to the ACI 318, the EC2 seems to overestimate the average flexural strength by about $8 \%$. In addition, regression analysis of the CC data indicates that part of the RAC-100 test results don't fall within a 95\% confidence interval of a nonlinear regression curve fit of the $\mathrm{CC}$ database. This result indicates that the RAC-100 flexural strength is lower than the CC for mixes with compressive strength around 35MPa and higher.

\section{Modulus of elasticity}

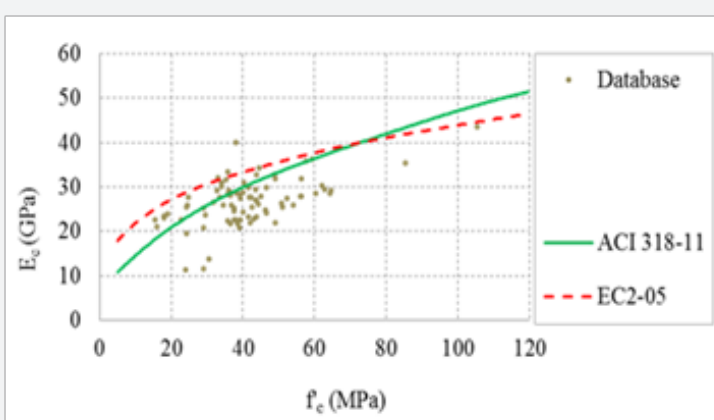

Figure 3: Database for modulus of elasticity of RAC.

Table 3 (a): Summary of modulus of elasticity test results.

\begin{tabular}{|c|c|c|c|c|c|c|c|c|c|c|c|c|c|}
\hline Author & Year & $\begin{array}{l}\text { RCA } \\
\text { (\%) }\end{array}$ & $f^{\prime} \mathbf{c}$ & Ec & $\begin{array}{l}\text { Test/ } \\
\text { ACI }\end{array}$ & $\begin{array}{l}\text { Test/ } \\
\text { EC }\end{array}$ & Author & Year & $\begin{array}{l}\text { RCA } \\
\text { (\%) }\end{array}$ & $f^{\prime} c$ & Ec & $\begin{array}{c}\text { Test/ } \\
\text { ACI }\end{array}$ & $\begin{array}{c}\text { Test/ } \\
\text { EC }\end{array}$ \\
\hline \multirow{3}{*}{ Katz [29] } & \multirow{3}{*}{2003} & \multirow{3}{*}{100} & 24.1 & 11.4 & 0.49 & 0.4 & \multirow{2}{*}{$\begin{array}{l}\text { Fathifazl } \\
\text { et al. [15] }\end{array}$} & 2009 & 63.5 & 24.1 & 11.4 & 0.98 & 0.88 \\
\hline & & & 30.5 & 13.7 & 0.53 & 0.45 & & & 74.3 & 30.5 & 13.7 & 0.97 & 0.9 \\
\hline & & & 29.1 & 11.5 & 0.45 & 0.38 & $\begin{array}{c}\text { Berndt } \\
{[34]}\end{array}$ & 2009 & 100 & 29.1 & 11.5 & 1.38 & 1.22 \\
\hline \multirow{10}{*}{$\begin{array}{c}\text { Ajdukiewicz \& } \\
\text { Kliszczewicz } \\
\text { [11] }\end{array}$} & \multirow{10}{*}{2007} & \multirow{10}{*}{100} & 34.6 & 25.9 & 0.94 & 0.81 & $\begin{array}{l}\text { Debieb et } \\
\text { al. [35] }\end{array}$ & 2010 & 100 & 34.6 & 25.9 & 0.85 & 0.68 \\
\hline & & & 56.4 & 31.8 & 0.9 & 0.86 & \multirow{3}{*}{$\begin{array}{c}\text { Bai \& Sun } \\
{[36]}\end{array}$} & 2010 & 50 & 56.4 & 31.8 & 0.91 & 0.82 \\
\hline & & & 40.1 & 24.3 & 0.82 & 0.73 & & & 70 & 40.1 & 24.3 & 0.87 & 0.79 \\
\hline & & & 60.2 & 28.5 & 0.78 & 0.76 & & & 100 & 60.2 & 28.5 & 0.87 & 0.79 \\
\hline & & & 85.3 & 35.3 & 0.81 & 0.84 & \multirow{3}{*}{$\begin{array}{c}\text { Choi et al. } \\
\text { [16] }\end{array}$} & 2010 & 30 & 85.3 & 35.3 & 1.18 & 0.96 \\
\hline & & & 35.3 & 31.7 & 1.14 & 0.99 & & & 50 & 35.3 & 31.7 & 1.1 & 0.89 \\
\hline & & & 57.6 & 35.9 & 1.01 & 0.97 & & & 100 & 57.6 & 35.9 & 1.01 & 0.8 \\
\hline & & & 105.3 & 43.5 & 0.9 & 0.98 & \multirow{3}{*}{$\begin{array}{l}\text { Zega \& Di } \\
\text { Maio [37] }\end{array}$} & 2010 & 25 & 105.3 & 43.5 & 1.17 & 0.9 \\
\hline & & & 36.6 & 28.1 & 0.99 & 0.87 & & & & 36.6 & 28.1 & 1.19 & 1.02 \\
\hline & & & 35.8 & 33.4 & 1.19 & 1.04 & & & 50 & 35.8 & 33.4 & 1.15 & 0.88 \\
\hline
\end{tabular}




\section{Civil Engineering Research Journal}

\begin{tabular}{|c|c|c|c|c|c|c|c|c|c|c|c|c|c|}
\hline $\begin{array}{l}\text { Etxeberria et } \\
\text { al. [10] }\end{array}$ & 2007 & 100 & 38.3 & 28.64 & 0.98 & 0.87 & & & & 38.3 & 28.6 & 1.13 & 0.98 \\
\hline \multirow{2}{*}{ Yang et al. [13] } & \multirow{2}{*}{2008} & \multirow{2}{*}{100} & 36 & 29.22 & 1.04 & 0.9 & & & \multirow{2}{*}{75} & 36 & 29.2 & 1.12 & 0.83 \\
\hline & & & 29.5 & 23.72 & 0.93 & 0.78 & & & & 29.5 & 23.7 & 1.09 & 0.94 \\
\hline \multirow{6}{*}{$\begin{array}{c}\text { Casuccio et al. } \\
\text { [33] }\end{array}$} & \multirow{6}{*}{2008} & \multirow{6}{*}{100} & 18 & 23.4 & 1.17 & 0.89 & \multirow{6}{*}{$\begin{array}{l}\text { Butler et } \\
\text { al. [21] }\end{array}$} & \multirow{6}{*}{2013} & \multirow{6}{*}{100} & 18 & 23.4 & 0.93 & 0.86 \\
\hline & & & 15.4 & 22.6 & 1.23 & 0.9 & & & & 15.4 & 22.6 & 0.93 & 0.83 \\
\hline & & & 36.4 & 28.8 & 1.02 & 0.89 & & & & 36.4 & 28.8 & 0.88 & 0.8 \\
\hline & & & 35.7 & 28.3 & 1.01 & 0.88 & & & & 35.7 & 28.3 & 0.82 & 0.8 \\
\hline & & & 44.4 & 34.3 & 1.1 & 1 & & & & 44.4 & 34.3 & 0.77 & 0.76 \\
\hline & & & 43.8 & 32.7 & 1.05 & 0.95 & & & & 43.8 & 32.7 & 0.76 & 0.74 \\
\hline
\end{tabular}

Table 3 (b): Summary of modulus of elasticity test results.

\begin{tabular}{|c|c|c|c|c|c|c|c|c|c|c|c|c|c|}
\hline Author & Year & $\begin{array}{l}\text { RCA } \\
\text { (\%) }\end{array}$ & $f^{\prime} c$ & Ec & $\begin{array}{c}\text { Test/ } \\
\text { ACI }\end{array}$ & $\begin{array}{l}\text { Test/ } \\
\text { EC }\end{array}$ & Author & Year & $\begin{array}{l}\text { RCA } \\
\text { (\%) }\end{array}$ & $\mathbf{f}^{\prime} \mathbf{c}$ & Ec & $\begin{array}{c}\text { Test/ } \\
\text { ACI }\end{array}$ & $\begin{array}{l}\text { Test/ } \\
\text { EC }\end{array}$ \\
\hline \multirow{10}{*}{ Kou et al. [38] } & \multirow{10}{*}{2011} & \multirow{10}{*}{100} & 38.1 & 22.5 & 0.78 & 0.68 & \multirow{17}{*}{$\begin{array}{c}\text { Knaack \& } \\
\text { Kurama } \\
\text { [32] }\end{array}$} & 2014 & 50 & 40 & 28.8 & 0.97 & 0.86 \\
\hline & & & 36.5 & 21.6 & 0.76 & 0.67 & & & 50 & 39.3 & 28.3 & 0.96 & 0.85 \\
\hline & & & 46.6 & 24.1 & 0.75 & 0.69 & & & 100 & 43.8 & 23.3 & 0.75 & 0.68 \\
\hline & & & 43.1 & 22.8 & 0.74 & 0.67 & & & 100 & 38.5 & 21.6 & 0.74 & 0.66 \\
\hline & & & 51.1 & 25.4 & 0.76 & 0.71 & & & 50 & 43.6 & 29.4 & 0.95 & 0.86 \\
\hline & & & 46.2 & 24.9 & 0.78 & 0.72 & & & 50 & 40.2 & 31 & 1.04 & 0.93 \\
\hline & & & 56.3 & 27.8 & 0.79 & 0.75 & & & 100 & 41.4 & 23.3 & 0.77 & 0.69 \\
\hline & & & 50.8 & 26.3 & 0.79 & 0.73 & & & 100 & 35.7 & 22.3 & 0.79 & 0.69 \\
\hline & & & 62.7 & 29.5 & 0.79 & 0.77 & & & 50 & 36.7 & 26 & 0.91 & 0.8 \\
\hline & & & 52.2 & 27.4 & 0.81 & 0.76 & & & 50 & 37.6 & 24.6 & 0.85 & 0.75 \\
\hline \multirow{3}{*}{$\begin{array}{c}\text { Ignjatovic et al. } \\
{[20]}\end{array}$} & \multirow{3}{*}{2013} & 50 & 44.2 & 26.2 & 0.84 & 0.76 & & & 100 & 42 & 21.9 & 0.72 & 0.65 \\
\hline & & 100 & & & & & & & & & & & \\
\hline & & 100 & 42.5 & 25.4 & 0.83 & 0.75 & & & 100 & 37.5 & 22.5 & 0.78 & 0.69 \\
\hline $\begin{array}{c}\text { Folino \& Xargay } \\
\text { [26] }\end{array}$ & 2014 & 100 & 29.1 & 20.75 & 0.82 & 0.68 & & & 50 & 41.8 & 27.6 & 0.91 & 0.82 \\
\hline \multirow{3}{*}{ Cui et al. [39] } & \multirow{3}{*}{2014} & \multirow{3}{*}{100} & 49 & 22 & 0.67 & 0.62 & & & 50 & 37.4 & 25.2 & 0.88 & 0.77 \\
\hline & & & 56 & 27.8 & 0.79 & 0.75 & & & 100 & 39.1 & 20.9 & 0.71 & 0.63 \\
\hline & & & 54 & 25.9 & 0.75 & 0.71 & & & 100 & 39.2 & 22.7 & 0.77 & 0.68 \\
\hline \multirow{5}{*}{ Kang et al. [25] } & \multirow{5}{*}{2014} & 15 & 59.4 & 36.2 & 1 & 0.96 & \multirow{4}{*}{ Steele [28] } & 2014 & 50 & 24.5 & 25.9 & 1.11 & 0.9 \\
\hline & & 30 & 48.8 & 32.8 & 1 & 0.93 & & & 100 & 33.4 & 27.6 & 1.02 & 0.87 \\
\hline & & 15 & 32.7 & 29.2 & 1.09 & 0.93 & & & & & & & \\
\hline & & 30 & 31.7 & 26.5 & 1 & 0.85 & & & & & & & \\
\hline & & 50 & 29 & 25.3 & 1 & 0.84 & Ave. & & & & & 0.91 & 0.81 \\
\hline
\end{tabular}


Table $3(a, b)$ presents the test results for modulus of elasticity from literature on RAC with replacement level from $25 \%$ to $100 \%$. Comparison of test results with the ACI 318 [4] and EC 2 [6] provisions showed only $34 \%$ and $5 \%$ of results is higher than the codes predictions, respectively (Figure 3). Almost all the previous studies reported lower modulus of elasticity for RAC compared to CC and it may be attributed to the lower stiffness and rigidity of the RCA particles which in turn decrease the rigidity and modulus of elasticity of the RAC [8].

\section{Fracture energy}

Limited literature is available in the case of the fracture energy of RAC. Results presented in [21,24,27,33] suggest decrease in fracture energy as a function of increases in RCA replacement ratio. Table 4 shows that the CEB-FIP [5] code provision is conservative only for $7 \%$ of the investigated data points The Japan's code [7] underestimates the fracture energy for $17 \%$ of the experimental results (Table 4). Likewise splitting tensile strength, statistical analysis of the data shows that the RAC-100 test results fall within a $95 \%$ confidence interval of a nonlinear regression curve fit of the $\mathrm{CC}$ database. That means the RAC-100 fracture energy is similar to the CC (Figure 4).

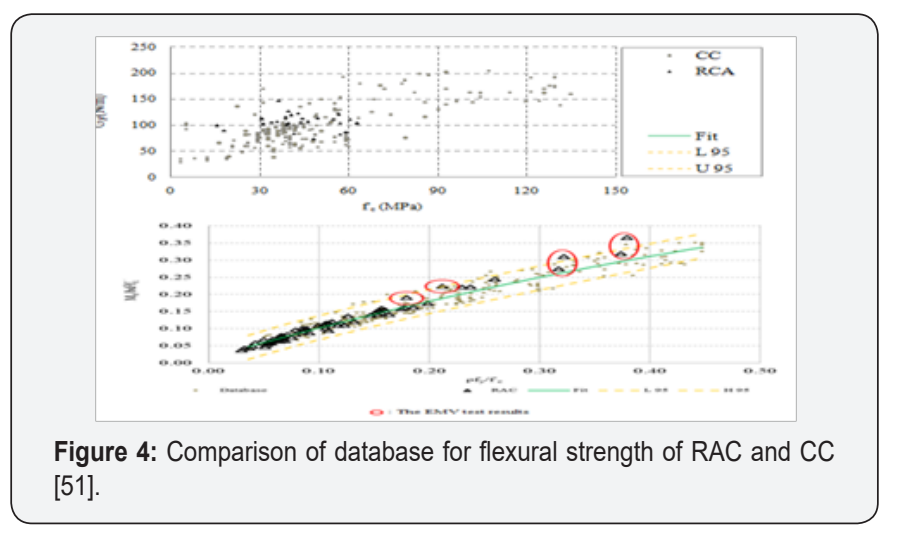

Table 4: Summary of fracture energy test results.

\begin{tabular}{|c|c|c|c|c|c|c|c|c|c|c|c|c|c|}
\hline Author & Year & $\begin{array}{l}\text { RCA } \\
\text { (\%) }\end{array}$ & $f^{\prime} c$ & GF & $\begin{array}{c}\text { Test/ } \\
\text { CEB- } \\
\text { FIP }\end{array}$ & $\begin{array}{l}\text { Test/ } \\
\text { JSCE }\end{array}$ & Author & Year & $\begin{array}{l}\text { RCA } \\
\text { (\%) }\end{array}$ & $f^{\prime} \mathbf{c}$ & Ec & $\begin{array}{c}\text { Test/ } \\
\text { CEB- } \\
\text { FIP }\end{array}$ & $\begin{array}{l}\text { Test/ } \\
\text { JSCE }\end{array}$ \\
\hline \multirow{6}{*}{$\begin{array}{l}\text { Casuccio } \\
\text { et al. [33] }\end{array}$} & \multirow{6}{*}{2008} & \multirow{6}{*}{100} & 18 & 90 & 0.73 & 1.13 & \multirow{8}{*}{ Butler et al. [27] } & \multirow{6}{*}{2014} & \multirow{6}{*}{100} & 30.7 & 112 & 0.83 & 1.37 \\
\hline & & & 15.4 & 99 & 0.83 & 1.31 & & & & 38.6 & 114.7 & 0.81 & 1.3 \\
\hline & & & 36.4 & 147 & 1.05 & 1.46 & & & & 48.2 & 73.8 & 0.5 & 0.78 \\
\hline & & & 35.7 & 107 & 0.77 & 1.07 & & & & 60.1 & 111.1 & 0.73 & 1.09 \\
\hline & & & 44.4 & 113 & 0.78 & 1.05 & & & & 31.1 & 102 & 0.75 & 1.24 \\
\hline & & & 43.8 & 106 & 0.74 & 0.99 & & & & 49.4 & 113.8 & 0.77 & 1.19 \\
\hline \multirow{11}{*}{$\begin{array}{l}\text { Butler et } \\
\text { al. [21] }\end{array}$} & \multirow{11}{*}{2013} & \multirow{11}{*}{100} & 46 & 106.3 & 0.73 & 1.14 & & & & 39.3 & 101.7 & 0.72 & 1.15 \\
\hline & & & 42.8 & 123.1 & 0.86 & 1.35 & & & & 62.8 & 103.7 & 0.67 & 1 \\
\hline & & & 39.7 & 126.7 & 0.89 & 1.42 & \multirow{8}{*}{$\begin{array}{l}\text { Arezoumandi et } \\
\text { al. [24] }\end{array}$} & 2014 & 30 & 44.5 & 148.9 & 1.03 & 1.47 \\
\hline & & & 39.7 & 104.2 & 0.74 & 1.17 & & & 50 & 45.5 & 143.5 & 0.99 & 1.41 \\
\hline & & & 40.3 & 119.1 & 0.84 & 1.33 & & & 70 & 35.2 & 123 & 0.89 & 1.31 \\
\hline & & & 41.2 & 84.2 & 0.59 & 0.93 & & & 100 & 34.1 & 105.9 & 0.77 & 1.14 \\
\hline & & & 54.7 & 111.1 & 0.74 & 1.12 & & & & & & & \\
\hline & & & 56.8 & 103.8 & 0.69 & 1.04 & & & & & & & \\
\hline & & & 56.8 & 82.3 & 0.54 & 0.82 & & & & & & & \\
\hline & & & 56.4 & 120.7 & 0.8 & 1.21 & & & & & & & \\
\hline & & & 59 & 86.7 & 0.57 & 0.85 & Ave. & & & & & 0.77 & 1.17 \\
\hline
\end{tabular}

\section{Structural Performance}

The following sections compare structural test results of RAC including bond, flexural, and shear strength with both codes provisions and CC databases.

\section{Bond Strength}

Three most common methods to measure bond strength between reinforcing steel and concrete are pull-out, beam-end, and splice specimens. Although pull-out test method is the most commonly used due to the ease of sample fabrication, but this method has the least realistic bond strength measurements. The reason is the bar at pull-out specimen is loaded in tension and the surrounding concrete is in compression, but in most practical applications, both the bar and surrounding concrete experience tensile stresses. For beam-end and splice specimens test methods, both the bar and surrounding concrete are in tension and provide a more realistic response [40]. The following section discusses the results of each test method separately.

\section{Pull-out}

Table 5(a-e) summarizes the results of pull-out tests from previous studies including RCA replacement level, compressive strength, $f_{c}^{\prime}$ and maximum aggregate size $\left(d_{\max }\right)$, rebar size, yielding strength of reinforcing steel $\left(f_{y}\right)$, average bond stress, $\mathrm{T}$, 
and normalized bond strength, $\tau / \sqrt{ }{f^{\prime}}_{c}$ and $\tau /{ }^{4} \sqrt{ } f^{\prime}{ }_{c}$. The bond strength provision is a function of the inverse square root of the compressive strength of concrete for most of design standards
$[4,7,14-16]$, but the ACI 408 [13] recommends the fourth root of the compressive strength of concrete instead of the square root. For this reason, Table 5(a-e) presents normalized bond strength of previous studies for both methods.

Table 5 (a): Summary of pull-out test results.

\begin{tabular}{|c|c|c|c|c|c|c|c|c|c|c|}
\hline Author & Year & Specimen & RCA (\%) & $d_{\max }(m m)$ & $\begin{array}{c}\text { Rebar } \\
\text { Size }(\mathrm{mm})\end{array}$ & $f_{y}$ & $\mathrm{f}_{\mathrm{c}}^{\prime}(\mathrm{MPa})$ & $\tau$ (MPa) & $\tau / \sqrt{\mathbf{f}^{\prime}}{ }^{\prime}$ & $\tau / 4 \sqrt{f^{\prime}}{ }_{c}$ \\
\hline \multirow{10}{*}{$\begin{array}{c}\text { Ajdukiewic \& } \\
\text { Kliszczewicz [9] }\end{array}$} & \multirow{10}{*}{2002} & R 5.1 & 0 & 16 & 14 & 410 & 48.4 & 29.1 & 4.2 & 11 \\
\hline & & R 8.1 & 0 & 16 & 14 & 410 & 48.9 & 29.1 & 4.2 & 11 \\
\hline & & R 9.1 & 0 & 16 & 14 & 410 & 48.9 & 29.1 & 4.2 & 11 \\
\hline & & R 11.1 & 0 & 16 & 14 & 410 & 52.3 & 26 & 3.6 & 9.7 \\
\hline & & Ave. & & & & & & & 4 & 10.7 \\
\hline & & R 5.2 & 100 & 16 & 14 & 410 & 44.5 & 27.3 & 4.1 & 10.6 \\
\hline & & R 8.2 & 100 & 16 & 14 & 410 & 45.2 & 26.8 & 4 & 10.3 \\
\hline & & R 9.2 & 100 & 16 & 14 & 410 & 49.6 & 26.4 & 3.7 & 9.9 \\
\hline & & R 11.2 & 100 & 16 & 14 & 410 & 54.4 & 24.4 & 3.3 & 9 \\
\hline & & Ave. & & & & & & & 3.8 & 10 \\
\hline \multirow{3}{*}{$\begin{array}{c}\text { Xiao \& Falkner } \\
{[43]}\end{array}$} & \multirow{3}{*}{2007} & RAC-II-0 & 0 & 13 & 10 & 420 & 43.5 & 17.4 & 2.6 & 6.8 \\
\hline & & RAC-II-50 & 50 & 13 & 10 & 420 & 39.3 & 17.2 & 2.8 & 6.9 \\
\hline & & $\begin{array}{l}\text { RAC- } \\
\text { II-100 }\end{array}$ & 100 & 13 & 10 & 420 & 34.6 & 17.4 & 3 & 7.2 \\
\hline \multirow{6}{*}{$\begin{array}{c}\text { Breccolotti \& } \\
\text { Materazzi [44] }\end{array}$} & \multirow{6}{*}{2013} & $\mathrm{C} 0-0-1$ & 0 & 24 & 14 & $\mathrm{NR}^{*}$ & 55.8 & 25.2 & 3.4 & 9.2 \\
\hline & & C25-50-1 & 50 & 24 & 14 & $\mathrm{NR}^{*}$ & 47.2 & 20.1 & 2.9 & 7.7 \\
\hline & & C25-100-1 & 100 & 24 & 14 & $\mathrm{NR}^{*}$ & 36.5 & 22.7 & 3.8 & 9.2 \\
\hline & & $\mathrm{C} 0-0-2$ & 0 & 24 & 14 & $\mathrm{NR}^{*}$ & 53.1 & 25.5 & 3.5 & 9.4 \\
\hline & & C25-50-2 & 50 & 24 & 14 & $\mathrm{NR}^{*}$ & 50.6 & 25.9 & 3.6 & 9.7 \\
\hline & & C25-100-2 & 100 & 24 & 14 & $\mathrm{NR}^{*}$ & 45.1 & 24 & 3.6 & 9.3 \\
\hline \multirow{5}{*}{ Kim \& Yun [22] } & \multirow{5}{*}{2013} & $\begin{array}{c}\text { RCA- } \\
\text { IOV-28 }\end{array}$ & 0 & 20 & 16 & 383 & 37 & 27.6 & 4.5 & 11.2 \\
\hline & & $\begin{array}{c}\text { RCA- } \\
\text { I30V-28 }\end{array}$ & 30 & 20 & 16 & 383 & 33.8 & 26.5 & 4.6 & 11 \\
\hline & & $\begin{array}{c}\text { RCA- } \\
\text { I60V-28 }\end{array}$ & 60 & 20 & 16 & 383 & 32.4 & 28.3 & 5 & 11.9 \\
\hline & & $\begin{array}{c}\text { RCA- } \\
\text { I100V-28 }\end{array}$ & 100 & 20 & 16 & 383 & 29.2 & 26.9 & 5 & 11.6 \\
\hline & & $\begin{array}{c}\text { RCA- } \\
\text { I0V-365 }\end{array}$ & 0 & 20 & 16 & 383 & 50.6 & 29.9 & 4.2 & 11.2 \\
\hline
\end{tabular}

Table 5 (b): Summary of pull-out test results.

\begin{tabular}{|c|c|c|c|c|c|c|c|c|c|c|}
\hline Author & Year & Specimen & RCA (\%) & $\mathbf{d}_{\max }$ & $\begin{array}{c}\text { Rebar } \\
\text { Size }\end{array}$ & $f_{y}$ & $f_{c}$ & $\tau$ & $\tau / \sqrt{f_{c}}$ & $\tau / 4 \sqrt{f_{c}}$ \\
\hline \multirow{6}{*}{$\begin{array}{c}\text { Kim \& Yun } \\
{[22]}\end{array}$} & \multirow{6}{*}{2013} & $\begin{array}{c}\text { RCA- } \\
\text { I30V-365 }\end{array}$ & 30 & 20 & 16 & 383 & 46.9 & 30 & 4.4 & 11.5 \\
\hline & & $\begin{array}{c}\text { RCA- } \\
\text { I60V-365 }\end{array}$ & 60 & 20 & 16 & 383 & 47.2 & 30.6 & 4.5 & 11.7 \\
\hline & & $\begin{array}{c}\text { RCA- } \\
\text { I100V-365 }\end{array}$ & 100 & 20 & 16 & 383 & 42.7 & 29.9 & 4.6 & 11.7 \\
\hline & & $\begin{array}{c}\text { RCA- } \\
\text { I0V-730 }\end{array}$ & 0 & 20 & 16 & 383 & 47.7 & 23.6 & 3.4 & 9 \\
\hline & & $\begin{array}{c}\text { RCA- } \\
\text { I30V-730 }\end{array}$ & 30 & 20 & 16 & 383 & 43.1 & 23.1 & 3.5 & 9 \\
\hline & & $\begin{array}{c}\text { RCA- } \\
\text { I60V-730 }\end{array}$ & 60 & 20 & 16 & 383 & 45.8 & 23.9 & 3.5 & 9.2 \\
\hline
\end{tabular}




\section{Civil Engineering Research Journal}

\begin{tabular}{|c|c|c|c|c|c|c|c|c|}
\hline $\begin{array}{c}\text { RCA- } \\
\text { I100V-730 }\end{array}$ & 100 & 20 & 16 & 383 & 37 & 21.7 & 3.6 & 8.8 \\
\hline RCA-IOV & 0 & 20 & 16 & 383 & 27.6 & 21.8 & 4.2 & 9.5 \\
\hline RCA-I30V & 30 & 20 & 16 & 383 & 26.5 & 22.8 & 4.4 & 10.1 \\
\hline RCA-I60V & 60 & 20 & 16 & 383 & 28.3 & 23.8 & 4.5 & 10.3 \\
\hline RCA-I100V & 100 & 20 & 16 & 383 & 26.9 & 24.8 & 4.8 & 10.9 \\
\hline RCA-IIOV & 0 & 25 & 16 & 383 & 25.3 & 25.8 & 5.1 & 11.5 \\
\hline RCA-II30V & 30 & 25 & 16 & 383 & 21.9 & 26.8 & 5.7 & 12.4 \\
\hline RCA-II60V & 60 & 25 & 16 & 383 & 21.6 & 27.8 & 6 & 12.9 \\
\hline $\begin{array}{l}\text { RCA- } \\
\text { II100V }\end{array}$ & 100 & 25 & 16 & 383 & 20.8 & 28.8 & 6.3 & 13.5 \\
\hline $\begin{array}{c}\text { RCA- } \\
\text { I0HB-28 }\end{array}$ & 0 & 20 & 16 & 383 & 37 & 16.8 & 2.8 & 6.8 \\
\hline $\begin{array}{c}\text { RCA- } \\
\text { I0HT-28 }\end{array}$ & 0 & 20 & 16 & 383 & 37 & 11.6 & 1.9 & 4.7 \\
\hline $\begin{array}{c}\text { RCA- } \\
\text { I30HB-28 }\end{array}$ & 30 & 20 & 16 & 383 & 33.8 & 22.6 & 3.9 & 9.4 \\
\hline $\begin{array}{c}\text { RCA- } \\
\text { I30HT-28 }\end{array}$ & 30 & 20 & 16 & 383 & 33.8 & 13 & 2.2 & 5.4 \\
\hline $\begin{array}{c}\text { RCA- } \\
\text { I60HB-28 }\end{array}$ & 60 & 20 & 16 & 383 & 32.4 & 24.4 & 4.3 & 10.2 \\
\hline $\begin{array}{c}\text { RCA- } \\
\text { I60HT-28 }\end{array}$ & 60 & 20 & 16 & 383 & 32.4 & 13.8 & 2.4 & 5.8 \\
\hline $\begin{array}{c}\text { RCA- } \\
\text { I100HB-28 }\end{array}$ & 100 & 20 & 16 & 383 & 29.2 & 24.7 & 4.6 & 10.6 \\
\hline $\begin{array}{c}\text { RCA- } \\
\text { I100HT-28 }\end{array}$ & 100 & 20 & 16 & 383 & 29.2 & 6.8 & 1.3 & 2.9 \\
\hline $\begin{array}{c}\text { RCA- } \\
\text { I0HB-365 }\end{array}$ & 0 & 20 & 16 & 383 & 50.6 & 20.3 & 2.9 & 7.6 \\
\hline $\begin{array}{c}\text { RCA- } \\
\text { I0HT-365 }\end{array}$ & 0 & 20 & 16 & 383 & 50.6 & 12.2 & 1.7 & 4.6 \\
\hline $\begin{array}{c}\text { RCA- } \\
\text { I30HB-365 }\end{array}$ & 30 & 20 & 16 & 383 & 46.9 & 26.4 & 3.9 & 10.1 \\
\hline $\begin{array}{c}\text { RCA- } \\
\text { I30HT-365 }\end{array}$ & 30 & 20 & 16 & 383 & 46.9 & 19.8 & 2.9 & 7.6 \\
\hline
\end{tabular}

Table 5 (c): Summary of pull-out test results.

\begin{tabular}{|c|c|c|c|c|c|c|c|c|c|c|}
\hline Author & Year & Specimen & RCA (\%) & $\mathbf{d}_{\max }$ & $\begin{array}{c}\text { Rebar } \\
\text { Size }\end{array}$ & $\mathbf{f y}$ & $\mathbf{f c}$ & $\boldsymbol{\tau}$ & $\boldsymbol{\tau} / \sqrt{\mathbf{f c}}$ & $\boldsymbol{\tau} / \mathbf{4} \sqrt{\mathbf{f c}}$ \\
\hline $\begin{array}{c}\text { Kim \& Yun } \\
{[22]}\end{array}$ & 2013 & RCA-I60HB-365 & 60 & 20 & 16 & 383 & 47.2 & 24.2 & 3.5 & 9.2 \\
\hline & & RCA-I60HT-365 & 60 & 20 & 16 & 383 & 47.2 & 18.6 & 2.7 & 7.1 \\
\hline & & RCA-I100HB-365 & 100 & 20 & 16 & 383 & 42.7 & 20.8 & 3.2 & 8.1 \\
\hline & & RCA-I100HT-365 & 100 & 20 & 16 & 383 & 42.7 & 12 & 1.8 & 4.7 \\
\hline & & RCA-I0HB-730 & 0 & 20 & 16 & 383 & 47.7 & 14.2 & 2.1 & 5.4 \\
\hline & RCA-I0HT-730 & 0 & 20 & 16 & 383 & 47.7 & 13 & 1.9 & 4.9 \\
\hline & & RCA-I30HB-730 & 30 & 20 & 16 & 383 & 43.1 & 15.8 & 2.4 & 6.2 \\
\hline & & RCA-I30HT-730 & 30 & 20 & 16 & 383 & 43.1 & 13 & 2 & 5.1 \\
\hline & & RCA-I60HB-730 & 60 & 20 & 16 & 383 & 45.8 & 21.9 & 3.2 & 8.4 \\
\hline & & RCA-I60HT-730 & 60 & 20 & 16 & 383 & 45.8 & 13.3 & 2 & 5.1 \\
\hline & & RCA-I100HB-730 & 100 & 20 & 16 & 383 & 37 & 22.1 & 3.6 & 9 \\
\hline & & RCA-I100HT-730 & 100 & 20 & 16 & 383 & 37 & 8.4 & 1.4 & 3.4 \\
\hline & & RCA-II0HB & 0 & 25 & 16 & 383 & 33.4 & 21.5 & 3.7 & 8.9 \\
\hline
\end{tabular}




\section{Civil Engineering Research Journal}

\begin{tabular}{|c|c|c|c|c|c|c|c|c|c|c|}
\hline & & RCA-IOHT & 0 & 25 & 16 & 383 & 33.4 & 13 & 2.2 & 5.4 \\
\hline & & RCA-II30HB & 30 & 25 & 16 & 383 & 31.5 & 17.2 & 3.1 & 7.3 \\
\hline & & RCA-II30HT & 30 & 25 & 16 & 383 & 31.5 & 11.2 & 2 & 4.7 \\
\hline & & RCA-II60HB & 60 & 25 & 16 & 383 & 30.7 & 19.8 & 3.6 & 8.4 \\
\hline & & RCA-II60HT & 60 & 25 & 16 & 383 & 30.7 & 9.8 & 1.8 & 4.2 \\
\hline & & RCA-II100HB & 100 & 25 & 16 & 383 & 29.5 & 17.4 & 3.2 & 7.5 \\
\hline & & RCA-II100HT & 100 & 25 & 16 & 383 & 29.5 & 9.1 & 1.7 & 3.9 \\
\hline \multirow[t]{7}{*}{ Steele [29] } & 2014 & VAC-P04-1 & 0 & 19 & 13 & 510 & 27.6 & 18.1 & 3.4 & 7.9 \\
\hline & & VAC-P04-2 & 0 & 19 & 13 & 510 & 27.6 & 18.3 & 3.5 & 8 \\
\hline & & VAC-P04-3 & 0 & 19 & 13 & 510 & 27.6 & 20 & 3.8 & 8.7 \\
\hline & & Ave. & & & & & & & 3.6 & 8.2 \\
\hline & & VAC-P06-1 & 0 & 19 & 19 & 516 & 27.6 & 21.2 & 4 & 9.2 \\
\hline & & VAC-P06-2 & 0 & 19 & 19 & 516 & 27.6 & 20.2 & 3.8 & 8.8 \\
\hline & & VAC-P06-3 & 0 & 19 & 19 & 516 & 27.6 & 19.9 & 3.8 & 8.7 \\
\hline
\end{tabular}

Table 5 (d): Summary of pull-out test results.

\begin{tabular}{|c|c|c|c|c|c|c|c|c|c|c|}
\hline Author & Year & Specimen & RCA (\%) & $d_{\max }$ & $\begin{array}{l}\text { Rebar } \\
\text { Size }\end{array}$ & fy & fc & $\tau$ & $\tau / \sqrt{\mathbf{f c}}$ & $\tau / \sqrt[4]{\mathrm{fc}}$ \\
\hline \multirow[t]{17}{*}{ Steele [29] } & 2014 & Ave. & & & & & & & 3.9 & 8.9 \\
\hline & & RAC50-P04-1 & 50 & 19 & 13 & 510 & 37.6 & 22.4 & 3.7 & 9 \\
\hline & & RAC50-P04-2 & 50 & 19 & 13 & 510 & 37.6 & 23 & 3.8 & 9.3 \\
\hline & & RAC50-P04-3 & 50 & 19 & 13 & 510 & 37.6 & 20.4 & 3.3 & 8.2 \\
\hline & & Ave. & & & & & & & 3.6 & 8.9 \\
\hline & & RAC50-P06-1 & 50 & 19 & 19 & 516 & 37.6 & 24.3 & 4 & 9.8 \\
\hline & & RAC50-P06-2 & 50 & 19 & 19 & 516 & 37.6 & 22.2 & 3.6 & 9 \\
\hline & & RAC50-P06-3 & 50 & 19 & 19 & 516 & 37.6 & 24.5 & 4 & 9.9 \\
\hline & & Ave. & & & & & & & 3.9 & 9.6 \\
\hline & & RAC100-P04-1 & 100 & 19 & 13 & 510 & 35.5 & 24.5 & 4.1 & 10 \\
\hline & & RAC100-P04-2 & 100 & 19 & 13 & 510 & 35.5 & 21.5 & 3.6 & 8.8 \\
\hline & & RAC100-P04-3 & 100 & 19 & 13 & 510 & 35.5 & 21.8 & 3.7 & 8.9 \\
\hline & & Ave. & & & & & & & 3.8 & 9.3 \\
\hline & & RAC100-P06-1 & 100 & 19 & 19 & 516 & 35.5 & 23.6 & 4 & 9.7 \\
\hline & & RAC100-P06-2 & 100 & 19 & 19 & 516 & 35.5 & 23.1 & 3.9 & 9.5 \\
\hline & & RAC100-P06-3 & 100 & 19 & 19 & 516 & 35.5 & 23.2 & 3.9 & 9.5 \\
\hline & & Ave. & & & & & & & 3.9 & 9.5 \\
\hline \multirow[t]{11}{*}{$\begin{array}{c}\text { Prince \& } \\
\text { Singh [46] }\end{array}$} & 2014 & A8R0-1 & 0 & 13 & 8 & 500 & 36.9 & 25.1 & 4.1 & 10.2 \\
\hline & & A8R0-2 & 0 & 13 & 8 & 500 & 36.9 & 22.7 & 3.7 & 9.2 \\
\hline & & A8R0-3 & 0 & 13 & 8 & 500 & 36.9 & 22.2 & 3.7 & 9 \\
\hline & & Ave. & & & & & & & 3.8 & 9.5 \\
\hline & & A8R25-1 & 25 & 13 & 8 & 500 & 28.9 & 15.2 & 2.8 & 6.6 \\
\hline & & A8R25-2 & 25 & 13 & 8 & 500 & 28.9 & 13.7 & 2.5 & 5.9 \\
\hline & & A8R25-3 & 25 & 13 & 8 & 500 & 28.9 & 13 & 2.4 & 5.6 \\
\hline & & Ave. & & & & & & & 2.6 & 6 \\
\hline & & A8R50-1 & 50 & 13 & 8 & 500 & 24 & 20.9 & 4.3 & 9.4 \\
\hline & & A8R50-2 & 50 & 13 & 8 & 500 & 24 & 20 & 4.1 & 9 \\
\hline & & A8R50-3 & 50 & 13 & 8 & 500 & 24 & 22.6 & 4.6 & 10.2 \\
\hline
\end{tabular}


Table 5 (e): Summary of pull-out test results.

\begin{tabular}{|c|c|c|c|c|c|c|c|c|c|c|}
\hline Author & Year & Specimen & RCA (\%) & $\mathbf{d}_{\max }$ & $\begin{array}{c}\text { Rebar } \\
\text { Size }\end{array}$ & fy & fc & $\tau$ & $\tau / \sqrt{\mathbf{f c}}$ & $\tau / 4 \sqrt{\mathrm{fc}}$ \\
\hline \multirow{30}{*}{$\begin{array}{c}\text { Prince \& } \\
\text { Singh [46] }\end{array}$} & \multirow{30}{*}{2014} & Ave. & & & & & & & 4.3 & 9.6 \\
\hline & & A8R75-1 & 75 & 13 & 8 & 500 & 26.2 & 16.7 & 3.3 & 7.4 \\
\hline & & A8R75-3 & 75 & 13 & 8 & 500 & 26.2 & 16.6 & 3.2 & 7.3 \\
\hline & & Ave. & & & & & & & 3.3 & 7.5 \\
\hline & & A8R100-1 & 100 & 13 & 8 & 500 & 24.7 & 21.7 & 4.4 & 9.7 \\
\hline & & A8R100-2 & 100 & 13 & 8 & 500 & 24.7 & 24 & 4.8 & 10.8 \\
\hline & & A8R100-3 & 100 & 13 & 8 & 500 & 24.7 & 22.5 & 4.5 & 10.1 \\
\hline & & Ave. & & & & & & & 4.6 & 10.2 \\
\hline & & A10R0-1 & 0 & 13 & 10 & 500 & 36.9 & 18.1 & 3 & 7.3 \\
\hline & & A10R0-2 & 0 & 13 & 10 & 500 & 36.9 & 17.6 & 2.9 & 7.1 \\
\hline & & A10R0-3 & 0 & 13 & 10 & 500 & 36.9 & 18.3 & 3 & 7.4 \\
\hline & & Ave. & & & & & & & 3 & 7.3 \\
\hline & & A10R25-1 & 25 & 13 & 10 & 500 & 28.9 & 18.5 & 3.4 & 8 \\
\hline & & A10R25-2 & 25 & 13 & 10 & 500 & 28.9 & 20 & 3.7 & 8.6 \\
\hline & & A10R25-3 & 25 & 13 & 10 & 500 & 28.9 & 19.3 & 3.6 & 8.3 \\
\hline & & Ave. & & & & & & & 3.6 & 8.3 \\
\hline & & A10R50-1 & 50 & 13 & 10 & 500 & 24 & 15.6 & 3.2 & 7 \\
\hline & & A10R50-2 & 50 & 13 & 10 & 500 & 24 & 18.6 & 3.8 & 8.4 \\
\hline & & A10R50-3 & 50 & 13 & 10 & 500 & 24 & 17.7 & 3.6 & 8 \\
\hline & & Ave. & & & & & & & 3.5 & 7.8 \\
\hline & & A10R75-1 & 75 & 13 & 10 & 500 & 26.2 & 15.7 & 3.1 & 6.9 \\
\hline & & A10R75-2 & 75 & 13 & 10 & 500 & 26.2 & 18.4 & 3.6 & 8.1 \\
\hline & & A10R75-3 & 75 & 13 & 10 & 500 & 26.2 & 18.7 & 3.7 & 8.3 \\
\hline & & Ave. & & & & & & & 3.4 & 7.8 \\
\hline & & $\begin{array}{c}\text { A10R100- } \\
1\end{array}$ & 100 & 13 & 10 & 500 & 24.7 & 18.4 & 3.7 & 8.3 \\
\hline & & $\begin{array}{c}\text { A10R100- } \\
2\end{array}$ & 100 & 13 & 10 & 500 & 24.7 & 19.2 & 3.9 & 8.6 \\
\hline & & $\begin{array}{c}\text { A10R100- } \\
3\end{array}$ & 100 & 13 & 10 & 500 & 24.7 & 17.3 & 3.5 & 7.8 \\
\hline & & Ave. & & & & & & & 3.7 & 8.2 \\
\hline & & A8R75-3 & 75 & 13 & 8 & 500 & 26.2 & 16.6 & 3.2 & 7.3 \\
\hline & & Ave. & & & & & & & 3.3 & 7.5 \\
\hline
\end{tabular}

Mukai \& Kikuchi [41] used both $15 \%$ and $30 \%$ RCA replacement in their specimens and reported no reduction in bond between reinforcing steel and RAC. Ajdukiewicz \& Kliszczewicz [11] replaced 100\% RCA from seven years old structures for pull-out specimens based on RILEM 7-II-128 [42]. Their results showed that the RAC specimens possess 7\% lower bond strength compared to the Xiao \& Falkner [43] tested 36 pull-out specimens with 0,50\%, and 100\% RCA replacement and reported similar load-slip curve for RAC specimens. However, $4 \%$ and $12 \%$ increase on bond strength of RAC was reported for the $50 \%$ and $100 \%$ replacements, respectively compared to the Breccolotti \& Materazzi [44] used pull-out specimens with 0,
$50 \%$, and $100 \%$ RCA replacement and observed no significant difference between bond strength of the RAC and CC specimens.

Kim \& Yun [22] used RCA with the replacement ratios of $30 \%, 60 \%$, and $100 \%$ to make 144 direct pull-out tests. Authors reported that the RAC specimens with the smaller aggregate size had a greater bond strength compared to the specimen with the larger aggregate size, while the CC specimen showed similar bond strength for both aggregate sizes. Furthermore, authors reported that the current ACI 318 [5] and CSA [45] codes provisions can be applied to RAC specimens. Steele [28] results showed that replacing both $50 \%$ and $100 \%$ RCA slightly improved the bond strength of reinforcing steel and RAC compared to the CC 


\section{Civil Engineering Research Journal}

specimens. Prince and Singh [46] tested 30 pull-out specimens with $0,25 \%, 50 \%, 75 \%$, and $100 \%$ RCA replacement ratios and reported identical load-slip relationships for the RAC and CC specimens. However, the bond strengths for $10 \mathrm{~mm}$ diameter deformed bars in the RAC were higher than the CC specimens.

\section{Splice Beam}

Table 6: presents dimensions and mechanical properties of materials used to test splice beams.

\begin{tabular}{|c|c|c|c|c|c|c|c|c|c|}
\hline Author & Year & Specimen & RCA (\%) & Rebar Size & $f_{c}$ & $f_{y}$ & $\mathbf{f}_{\mathrm{s}}$ & fs $/ \sqrt{\text { fc }}$ & $\mathrm{fs} / 4 \sqrt{\mathrm{fc}}$ \\
\hline \multirow{12}{*}{ Steele [29] } & \multirow{12}{*}{2014} & VAC-1 & 0 & 19 & 27.6 & 516 & 434.4 & 82.7 & 189.5 \\
\hline & & VAC-2 & 0 & 19 & 27.6 & 516 & 488.2 & 92.9 & 213 \\
\hline & & VAC-3 & 0 & 19 & 27.6 & 516 & 424.7 & 80.8 & 185.3 \\
\hline & & Ave. & & & & & & 85.5 & 195.9 \\
\hline & & RAC50-1 & 50 & 19 & 24.5 & 516 & 389.6 & 78.7 & 175.1 \\
\hline & & RAC50-2 & 50 & 19 & 24.5 & 516 & 380.6 & 76.9 & 171.1 \\
\hline & & RAC50-3 & 50 & 19 & 24.5 & 516 & 377.8 & 76.3 & 169.8 \\
\hline & & Ave. & & & & & & 77.3 & 172 \\
\hline & & RAC100-1 & 100 & 19 & 33.4 & 516 & 326.1 & 56.4 & 135.6 \\
\hline & & RAC100-2 & 100 & 19 & 33.4 & 516 & 344.1 & 59.5 & 143.1 \\
\hline & & RAC100-3 & 100 & 19 & 33.4 & 516 & 379.9 & 65.7 & 158 \\
\hline & & Ave. & & & & & & 60.6 & 145.6 \\
\hline Author & Year & Specimen & RCA (\%) & Rebar Size & fc & fy & fs & $\mathrm{fs} / \sqrt{\mathrm{fc}}$ & $\mathrm{fs} / 4 \sqrt{\mathrm{fc}}$ \\
\hline \multirow{12}{*}{ Steele [29] } & \multirow{12}{*}{2014} & VAC-1 & 0 & 19 & 27.6 & 516 & 434.4 & 82.7 & 189.5 \\
\hline & & VAC-2 & 0 & 19 & 27.6 & 516 & 488.2 & 92.9 & 213 \\
\hline & & VAC-3 & 0 & 19 & 27.6 & 516 & 424.7 & 80.8 & 185.3 \\
\hline & & Ave. & & & & & & 85.5 & 195.9 \\
\hline & & RAC50-1 & 50 & 19 & 24.5 & 516 & 389.6 & 78.7 & 175.1 \\
\hline & & RAC50-2 & 50 & 19 & 24.5 & 516 & 380.6 & 76.9 & 171.1 \\
\hline & & RAC50-3 & 50 & 19 & 24.5 & 516 & 377.8 & 76.3 & 169.8 \\
\hline & & Ave. & & & & & & 77.3 & 172 \\
\hline & & RAC100-1 & 100 & 19 & 33.4 & 516 & 326.1 & 56.4 & 135.6 \\
\hline & & RAC100-2 & 100 & 19 & 33.4 & 516 & 344.1 & 59.5 & 143.1 \\
\hline & & RAC100-3 & 100 & 19 & 33.4 & 516 & 379.9 & 65.7 & 158 \\
\hline & & Ave. & & & & & & 60.6 & 145.6 \\
\hline
\end{tabular}


Table 6 presents dimensions and mechanical properties of materials used to test splice beams. The only difference is that Table 6 includes reinforcing steel stress at failure, fs, instead of average bond stress based on ACI 408 [40] recommendation. Steele [28] tested full scale beams with cross section of $300 \times 460 \mathrm{~mm}$ for both $50 \%$ and $100 \%$ RCA replacement levels and reported that the bond strength decreased around $10 \%$ for $50 \%$ and $20 \%$ for $100 \%$ RCA replacement.

\section{End beam}

Fathifazl et al. [47] used both conventional method (replaced $100 \%$ RCA) as well as the equivalent mortar volume (EMV)

Table 7(a): Summary of end beam test results.

\begin{tabular}{|c|c|c|c|c|c|c|c|c|c|c|}
\hline Author & Year & Specimen & RCA (\%) & $\mathrm{d}_{\max }$ & Rebar Size & fy & fc & $\tau \mathrm{b}$ & $\tau / \sqrt{\mathrm{fc}}$ & $\tau / 4 \sqrt{\mathrm{fc}}$ \\
\hline \multirow{6}{*}{$\begin{array}{c}\text { Fathifazl } \\
\text { [47] }\end{array}$} & \multirow{6}{*}{2008} & CM-30 & 100 & 19 & 30 & 449 & 48.5 & 6.01 & 0.9 & 2.3 \\
\hline & & CL-30 & 0 & 19 & 30 & 449 & 38 & 6.95 & 1.1 & 2.8 \\
\hline & & CM-15 & 100 & 19 & 15 & 407 & 48.5 & 8.1 & 1.2 & 3.1 \\
\hline & & CV-30 & 100 & 19 & 30 & 449 & 49 & 6.29 & 0.9 & 2.4 \\
\hline & & EV-30 & 74.3 & 19 & 30 & 449 & 43.5 & 7.36 & 1.1 & 2.9 \\
\hline & & CG-30 & 0 & 19 & 30 & 449 & 35.9 & 7.13 & 1.2 & 2.9 \\
\hline \multirow{17}{*}{$\begin{array}{l}\text { Butler et } \\
\text { al. [27] }\end{array}$} & \multirow{17}{*}{2014} & $\begin{array}{l}\text { BE-NAC- } \\
30-125 A\end{array}$ & 0 & 19 & 25 & 467 & 34.5 & 6.66 & 1.1 & 2.7 \\
\hline & & $\begin{array}{l}\text { BE-NAC- } \\
30-125 B\end{array}$ & 0 & 19 & 25 & 467 & 34.5 & 7.55 & 1.3 & 3.1 \\
\hline & & $\begin{array}{l}\text { BE-NAC- } \\
40-125 A\end{array}$ & 0 & 19 & 25 & 467 & 40.5 & 6.21 & 1 & 2.5 \\
\hline & & $\begin{array}{l}\text { BE-NAC- } \\
40-125 B\end{array}$ & 0 & 19 & 25 & 467 & 39.4 & 6.92 & 1.1 & 2.8 \\
\hline & & $\begin{array}{l}\text { BE-NAC- } \\
50-125 A\end{array}$ & 0 & 19 & 25 & 467 & 49 & 6.81 & 1 & 2.6 \\
\hline & & $\begin{array}{l}\text { BE-NAC- } \\
50-125 B\end{array}$ & 0 & 19 & 25 & 467 & 49 & 6.91 & 1 & 2.6 \\
\hline & & $\begin{array}{l}\text { BE-NAC- } \\
60-125 A\end{array}$ & 0 & 19 & 25 & 467 & 53.8 & 6.84 & 0.9 & 2.5 \\
\hline & & $\begin{array}{l}\text { BE-NAC- } \\
60-125 B\end{array}$ & 0 & 19 & 25 & 467 & 53.8 & 7.27 & 1 & 2.7 \\
\hline & & $\begin{array}{c}\text { BE-RAC1- } \\
30-125 A\end{array}$ & 100 & 19 & 25 & 467 & 30.9 & 5.72 & 1 & 2.4 \\
\hline & & $\begin{array}{c}\text { BE-RAC1- } \\
30-125 B\end{array}$ & 100 & 19 & 25 & 467 & 30.9 & 5.78 & 1 & 2.4 \\
\hline & & $\begin{array}{c}\text { BE-RAC1- } \\
40-125 A\end{array}$ & 100 & 19 & 25 & 467 & 43.7 & 6.17 & 0.9 & 2.4 \\
\hline & & $\begin{array}{c}\text { BE-RAC1- } \\
40-125 B\end{array}$ & 100 & 19 & 25 & 467 & 42.6 & 7.01 & 1.1 & 2.7 \\
\hline & & $\begin{array}{c}\text { BE-RAC1- } \\
50-125 A\end{array}$ & 100 & 19 & 25 & 467 & 47.9 & 6.25 & 0.9 & 2.4 \\
\hline & & $\begin{array}{c}\text { BE-RAC1- } \\
50-125 B\end{array}$ & 100 & 19 & 25 & 467 & 47.9 & 5.89 & 0.9 & 2.2 \\
\hline & & $\begin{array}{c}\text { BE-RAC1- } \\
60-125 A\end{array}$ & 100 & 19 & 25 & 467 & 53.8 & 6.4 & 0.9 & 2.4 \\
\hline & & $\begin{array}{c}\text { BE-RAC1- } \\
60-125 B\end{array}$ & 100 & 19 & 25 & 467 & 49.9 & 6.96 & 1 & 2.6 \\
\hline & & $\begin{array}{c}\text { BE-RAC2- } \\
30-125 A\end{array}$ & 100 & 19 & 25 & 467 & 31.3 & 5.15 & 0.9 & 2.2 \\
\hline
\end{tabular}

method for proportioning RAC. They reported 24\% lower bond strength for the conventional method, while the EMV method bond strength was comparable (only $6 \%$ reduction) with the CC specimens. Butler et al. [17] tested 48 beam-end specimens with $100 \%$ RCA replacement from different resources, including regional sidewalk, curb, and gutter infrastructure, apron and terminal structures from Pearson International Airport in Toronto, Canada, and a concrete ready-mix plant. Their results showed $3 \%$ to $20 \%$ reduction in bond strength between reinforcing steel and the RAC compared to the CC beams. A summary of details for end beam tests can be found in Table 7 (a,b). 


\begin{tabular}{|c|c|c|c|c|c|c|c|c|c|}
\hline & & $\begin{array}{c}\text { BE-RAC2- } \\
30-125 B\end{array}$ & 100 & 19 & 25 & 467 & 31.3 & 6.02 & 1.1 \\
\cline { 3 - 8 } & $\begin{array}{c}\text { BE-RAC2- } \\
50-125 \mathrm{~A}\end{array}$ & 100 & 19 & 25 & 467 & 49.4 & 5.88 \\
\hline
\end{tabular}

Table 7 (b): Summary of end beam test results.

\begin{tabular}{|c|c|c|c|c|c|c|c|c|c|c|}
\hline Author & Year & Specimen & RCA (\%) & $d_{\max }$ & $\begin{array}{c}\text { Rebar } \\
\text { Size }\end{array}$ & fy & fc & $\tau \mathbf{b}$ & $\tau / \sqrt{\mathrm{fc}}$ & $\tau / 4 \sqrt{\mathrm{fc}}$ \\
\hline \multirow{23}{*}{$\begin{array}{l}\text { Butler et } \\
\text { al. [27] }\end{array}$} & \multirow{23}{*}{2014} & $\begin{array}{c}\text { BE-RAC2- } \\
50-125 B\end{array}$ & 100 & 19 & 25 & 467 & 49.4 & 6.03 & 0.9 & 2.3 \\
\hline & & $\begin{array}{c}\text { BE-RAC3- } \\
40-125 A\end{array}$ & 100 & 19 & 25 & 467 & 41.4 & 6.5 & 1 & 2.6 \\
\hline & & $\begin{array}{c}\text { BE-RAC3- } \\
\text { 40-125B }\end{array}$ & 100 & 19 & 25 & 467 & 41.5 & 5.62 & 0.9 & 2.2 \\
\hline & & $\begin{array}{c}\text { BE-RAC3- } \\
60-125 A\end{array}$ & 100 & 19 & 25 & 467 & 56.2 & 6.71 & 0.9 & 2.5 \\
\hline & & $\begin{array}{c}\text { BE-RAC3- } \\
60-125 B\end{array}$ & 100 & 19 & 25 & 467 & 57 & 6.51 & 0.9 & 2.4 \\
\hline & & $\begin{array}{l}\text { BE-NAC- } \\
30-375 A\end{array}$ & 0 & 19 & 25 & 467 & 30 & 5.77 & 1.1 & 2.5 \\
\hline & & $\begin{array}{l}\text { BE-NAC- } \\
30-375 B\end{array}$ & 0 & 19 & 25 & 467 & 30 & 5.79 & 1.1 & 2.5 \\
\hline & & $\begin{array}{l}\text { BE-NAC- } \\
50-375 A\end{array}$ & 0 & 19 & 25 & 467 & 29 & 6.09 & 1.1 & 2.6 \\
\hline & & $\begin{array}{l}\text { BE-NAC- } \\
50-375 B\end{array}$ & 0 & 19 & 25 & 467 & 29 & 5.8 & 1.1 & 2.5 \\
\hline & & $\begin{array}{c}\text { BE-RAC1- } \\
30-375 A\end{array}$ & 100 & 19 & 25 & 467 & 30 & 5.12 & 0.9 & 2.2 \\
\hline & & $\begin{array}{c}\text { BE-RAC1- } \\
30-375 B\end{array}$ & 100 & 19 & 25 & 467 & 30 & 5.13 & 0.9 & 2.2 \\
\hline & & $\begin{array}{c}\text { BE-RAC1- } \\
50-375 A\end{array}$ & 100 & 19 & 25 & 467 & 31 & 5.5 & 1 & 2.3 \\
\hline & & $\begin{array}{c}\text { BE-RAC1- } \\
\text { 40-375B }\end{array}$ & 100 & 19 & 25 & 467 & 31 & 5.18 & 0.9 & 2.2 \\
\hline & & $\begin{array}{c}\text { BE-RAC2- } \\
\text { 30-375A }\end{array}$ & 100 & 19 & 25 & 467 & 33 & 5.05 & 0.9 & 2.1 \\
\hline & & $\begin{array}{c}\text { BE-RAC2- } \\
30-375 B\end{array}$ & 100 & 19 & 25 & 467 & 33 & 5.11 & 0.9 & 2.1 \\
\hline & & $\begin{array}{c}\text { BE-RAC2- } \\
50-375 A\end{array}$ & 100 & 19 & 25 & 467 & 33 & 5.59 & 1 & 2.3 \\
\hline & & $\begin{array}{c}\text { BE-RAC2- } \\
40-375 B\end{array}$ & 100 & 19 & 25 & 467 & 33 & 5.21 & 0.9 & 2.2 \\
\hline & & $\begin{array}{l}\text { BE-NAC- } \\
40-450 A\end{array}$ & 0 & 19 & 25 & 467 & 29 & 5.81 & 1.1 & 2.5 \\
\hline & & $\begin{array}{l}\text { BE-NAC- } \\
40-450 B\end{array}$ & 0 & 19 & 25 & 467 & 29 & 5.11 & 0.9 & 2.2 \\
\hline & & $\begin{array}{l}\text { BE-NAC- } \\
60-450 A\end{array}$ & 0 & 19 & 25 & 467 & 29 & 5.64 & 1 & 2.4 \\
\hline & & $\begin{array}{l}\text { BE-NAC- } \\
60-450 B\end{array}$ & 0 & 19 & 25 & 467 & 30 & 5.86 & 1.1 & 2.5 \\
\hline & & $\begin{array}{c}\text { BE-RAC1- } \\
40-450 A\end{array}$ & 100 & 19 & 25 & 467 & 35 & 5.39 & 0.9 & 2.2 \\
\hline & & $\begin{array}{c}\text { BE-RAC1- } \\
\text { 40-450B }\end{array}$ & 100 & 19 & 25 & 467 & 35 & 5.36 & 0.9 & 2.2 \\
\hline
\end{tabular}




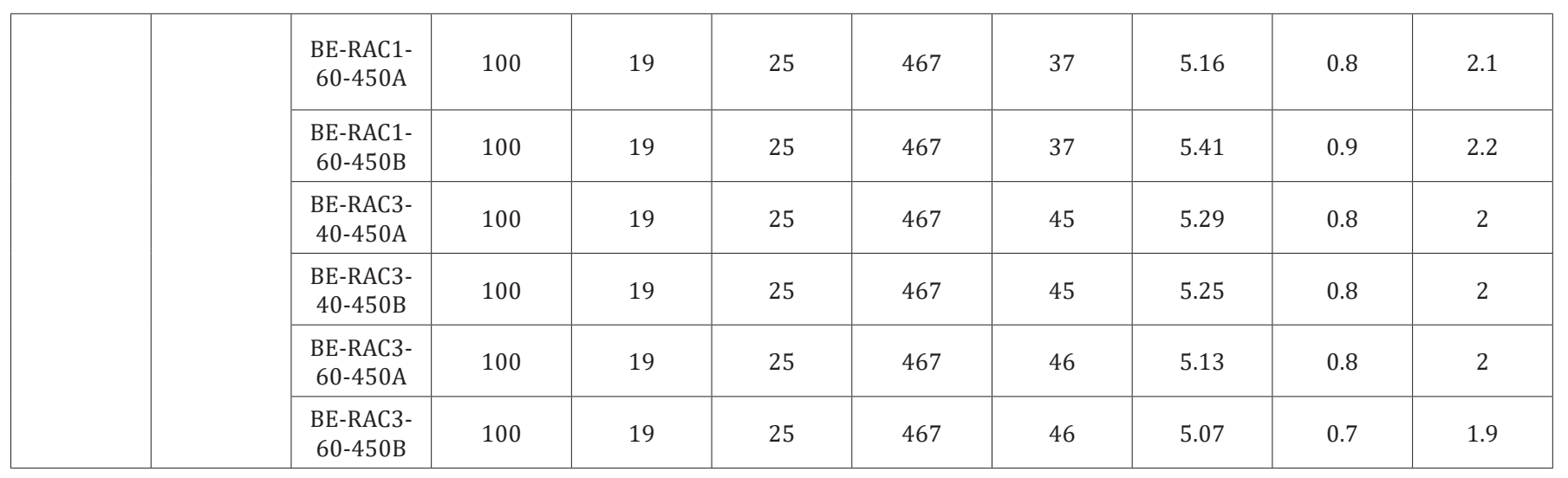

\section{Flexural Strength}

Table 8: Summary of flexural beam test results.

\begin{tabular}{|c|c|c|c|c|c|c|c|c|c|}
\hline Author & Year & Specimen & RCA (\%) & b & $\mathbf{h}$ & d & $\rho$ & fc & fy \\
\hline \multirow{3}{*}{$\begin{array}{l}\text { Yagishita et } \\
\text { al. [48] }\end{array}$} & \multirow{3}{*}{1994} & BR1 & 100 & 120 & 180 & 150 & 1.47 & 30.9 & 330 \\
\hline & & BR2 & 100 & 120 & 180 & 150 & 1.47 & 32 & 330 \\
\hline & & BR3 & 100 & 120 & 180 & 150 & 1.47 & 33.2 & 330 \\
\hline \multirow{3}{*}{$\begin{array}{l}\text { Maruyama } \\
\text { et al. [49] }\end{array}$} & \multirow{3}{*}{2004} & CRC30 & 100 & 150 & 200 & 160 & 1.11 & 69 & 331 \\
\hline & & CRC45 & 100 & 150 & 200 & 160 & 1.11 & 46.5 & 331 \\
\hline & & CRC60 & 100 & 150 & 200 & 160 & 1.11 & 32.9 & 331 \\
\hline \multirow{15}{*}{$\begin{array}{c}\text { Sato et al. } \\
\text { [12] }\end{array}$} & \multirow{15}{*}{2007} & $\begin{array}{l}\text { CR45-01- } \\
\text { 10WB }\end{array}$ & 100 & 150 & 200 & 160 & 0.59 & 30.4 & 332 \\
\hline & & $\begin{array}{l}\text { CR45-01- } \\
\text { 10DB }\end{array}$ & 100 & 150 & 200 & 160 & 0.59 & 28.4 & 332 \\
\hline & & $\begin{array}{l}\text { CR60-01- } \\
\text { 10WB }\end{array}$ & 100 & 150 & 200 & 160 & 0.59 & 34.5 & 332 \\
\hline & & $\begin{array}{l}\text { CR60-01- } \\
\text { 10DB }\end{array}$ & 100 & 150 & 200 & 160 & 0.59 & 31.8 & 332 \\
\hline & & $\begin{array}{l}\text { CR45-01- } \\
\text { 13WB }\end{array}$ & 100 & 150 & 200 & 160 & 1.06 & 30.4 & 353 \\
\hline & & $\begin{array}{l}\text { CR45-01- } \\
\text { 13DB }\end{array}$ & 100 & 150 & 200 & 160 & 1.06 & 28.4 & 353 \\
\hline & & $\begin{array}{l}\text { CR60-01- } \\
\text { 13WB }\end{array}$ & 100 & 150 & 200 & 160 & 1.06 & 34.5 & 353 \\
\hline & & $\begin{array}{l}\text { CR60-01- } \\
\text { 13DB }\end{array}$ & 100 & 150 & 200 & 160 & 1.06 & 31.8 & 353 \\
\hline & & $\begin{array}{l}\text { CR45-01- } \\
\text { 16WB }\end{array}$ & 100 & 150 & 200 & 160 & 1.65 & 30.4 & 342 \\
\hline & & $\begin{array}{c}\text { CR45-01- } \\
\text { 16DB }\end{array}$ & 100 & 150 & 200 & 160 & 1.65 & 28.4 & 342 \\
\hline & & $\begin{array}{l}\text { CR60-01- } \\
\text { 16WB }\end{array}$ & 100 & 150 & 200 & 160 & 1.65 & 34.5 & 342 \\
\hline & & $\begin{array}{l}\text { CR60-01- } \\
\text { 16DB }\end{array}$ & 100 & 150 & 200 & 160 & 1.65 & 31.8 & 342 \\
\hline & & CR30-03-DB & 100 & 150 & 200 & 160 & 1.06 & 69 & 331 \\
\hline & & CR45-03-DB & 100 & 150 & 200 & 160 & 1.06 & 46.5 & 331 \\
\hline & & CR60-03-DB & 100 & 150 & 200 & 160 & 1.06 & 32.9 & 331 \\
\hline
\end{tabular}


Table 8 (a): Summary of flexural beam test results.

\begin{tabular}{|c|c|c|c|c|c|c|c|c|c|}
\hline Author & Year & Specimen & RCA (\%) & b & h & d & $\rho$ & fc & fy \\
\hline \multirow{10}{*}{$\begin{array}{c}\text { Ajdukiewicz \& } \\
\text { Kliszczewicz [11] }\end{array}$} & \multirow{10}{*}{2007} & ORNl-b1 & 100 & 200 & 300 & 291 & 0.77 & 34.6 & 410 \\
\hline & & ORNm-b1 & 100 & 200 & 300 & 291 & 0.77 & 56.4 & 410 \\
\hline & & GRNl-b1 & 100 & 200 & 300 & 291 & 0.77 & 40.1 & 410 \\
\hline & & GRNm-b1 & 100 & 200 & 300 & 291 & 0.77 & 60.2 & 410 \\
\hline & & GRNh-b1 & 100 & 200 & 300 & 291 & 0.77 & 85.3 & 410 \\
\hline & & BRNl-b1 & 100 & 200 & 300 & 291 & 0.77 & 35.3 & 410 \\
\hline & & BRNm-b1 & 100 & 200 & 300 & 291 & 0.77 & 57.6 & 410 \\
\hline & & BRNh-b1 & 100 & 200 & 300 & 291 & 0.77 & 105.3 & 410 \\
\hline & & ORNl-b2 & 100 & 200 & 300 & 291 & 1.37 & 36.6 & 410 \\
\hline & & BRNl-b2 & 100 & 200 & 300 & 291 & 1.37 & 35.8 & 410 \\
\hline \multirow{5}{*}{ Bai \& Sun [36] } & \multirow{5}{*}{2010} & RAC-2 & 50 & 150 & 300 & 260 & 0.68 & 42.3 & 420 \\
\hline & & RAC-3 & 70 & 150 & 300 & 260 & 0.68 & 43.7 & 420 \\
\hline & & RAC-4 & 100 & 150 & 300 & 260 & 0.68 & 43.5 & 420 \\
\hline & & RAC-5 & 100 & 150 & 300 & 260 & 0.89 & 43.5 & 420 \\
\hline & & RAC-6 & 100 & 150 & 300 & 260 & 1.03 & 43.5 & 420 \\
\hline \multirow{8}{*}{ Fathifazl et al. [15] } & \multirow{8}{*}{2009} & EM-Min & 63.5 & 200 & 350 & 304 & 0.39 & 36.9 & 473 \\
\hline & & EM-Av & 63.5 & 200 & 350 & 301 & 1.92 & 36.9 & 407 \\
\hline & & EM-Max & 63.5 & 200 & 390 & 307 & 3.2 & 36.9 & 431 \\
\hline & & EM-CMP & 63.5 & 200 & 350 & 302 & 3.25 & 36.9 & 431 \\
\hline & & EV-Min & 74.3 & 200 & 350 & 304 & 0.39 & 43.5 & 473 \\
\hline & & EV-Av & 74.3 & 200 & 350 & 301 & 1.92 & 43.5 & 407 \\
\hline & & EV-Max & 74.3 & 200 & 390 & 307 & 3.2 & 43.5 & 431 \\
\hline & & EV-CMP & 74.3 & 200 & 350 & 302 & 3.25 & 43.5 & 431 \\
\hline \multirow{4}{*}{$\begin{array}{l}\text { Arezoumandi et al. } \\
\qquad[50]\end{array}$} & \multirow{4}{*}{2015} & F-6-1 & 100 & 305 & 460 & 400 & 0.47 & 30.5 & 568 \\
\hline & & F-6-2 & 100 & 305 & 460 & 400 & 0.47 & 31.3 & 568 \\
\hline & & F-7-1 & 100 & 305 & 460 & 400 & 0.64 & 30.5 & 517 \\
\hline & & F-7-2 & 100 & 305 & 460 & 400 & 0.64 & 31.3 & 517 \\
\hline
\end{tabular}

Table 8 (b): Summary of flexural beam test results.

\begin{tabular}{|c|c|c|c|c|c|c|c|c|c|}
\hline Author & Year & Specimen & RCA (\%) & b & h & d & $\rho$ & fc & fy \\
\hline \multirow[t]{13}{*}{$\begin{array}{c}\text { Kang et al. } \\
\text { [25] }\end{array}$} & 2014 & H15-0.5 & 15 & 135 & 270 & 230 & 0.51 & 59.4 & 377 \\
\hline & & H15-1.0 & 15 & 135 & 270 & 230 & 0.85 & 59.4 & 408 \\
\hline & & H15-1.5 & 15 & 135 & 270 & 230 & 1.3 & 59.4 & 389 \\
\hline & & H15-1.8 & 15 & 135 & 270 & 230 & 1.83 & 59.4 & 411 \\
\hline & & H30-0.5 & 30 & 135 & 270 & 230 & 0.51 & 48.8 & 377 \\
\hline & & Н30-1.0 & 30 & 135 & 270 & 230 & 0.85 & 48.8 & 408 \\
\hline & & Н30-1.5 & 30 & 135 & 270 & 230 & 1.3 & 48.8 & 389 \\
\hline & & Н30-1.8 & 30 & 135 & 270 & 230 & 1.83 & 48.8 & 411 \\
\hline & & N15-0.5 & 15 & 135 & 270 & 230 & 0.51 & 32.7 & 377 \\
\hline & & N15-1.0 & 15 & 135 & 270 & 230 & 0.85 & 32.7 & 408 \\
\hline & & N15-1.5 & 15 & 135 & 270 & 230 & 1.3 & 32.7 & 389 \\
\hline & & N15-1.8 & 15 & 135 & 270 & 230 & 1.83 & 32.7 & 411 \\
\hline & & N30-0.5 & 30 & 135 & 270 & 230 & 0.51 & 31.7 & 377 \\
\hline
\end{tabular}




\section{Civil Engineering Research Journal}

\begin{tabular}{|l|c|c|c|c|c|c|c|c|c|}
\hline & & N30-1.0 & 30 & 135 & 270 & 230 & 0.85 & 31.7 & 408 \\
\hline & N30-1.5 & 30 & 135 & 270 & 230 & 1.3 & 31.7 & 389 \\
\hline & N30-1.8 & 30 & 135 & 270 & 230 & 1.83 & 31.7 & 411 \\
\hline & & N50-0.5 & 50 & 135 & 270 & 230 & 0.51 & 29 & 377 \\
\hline & & N50-1.0 & 50 & 135 & 270 & 230 & 0.85 & 29 & 408 \\
\hline & & N50-1.5 & 50 & 135 & 270 & 230 & 1.3 & 29 & 389 \\
\hline & & N50-1.8 & 50 & 135 & 270 & 230 & 1.83 & 29 & 411 \\
\hline
\end{tabular}

Table $8(a, b)$ presents details of flexural test specimens from previous research. Table 9 (a,b) compares cracking moment $\left(M_{c r}\right)$, yielding moment $\left(M_{y}\right)$, and ultimate moment $\left(M_{n}\right)$, of the RAC beams with both the ACI 318 [5] and EC 2 [7] provisions. Mukai \& Kikuchi [41] tested beams measuring $150 \times 150 \mathrm{~mm}$ in cross section and 1.8-m in length with both $15 \%$ and $30 \%$ RCA replacement ratios and reported no significant difference in ultimate moment. However, authors reported lower cracking moment for RAC beams. Yagashita et al. [48] used low grade, medium grade, and high grade types of recycled aggregate with $100 \%$ replacement for determining the flexural strength of reinforced concrete beams. Their results showed using high grade RCA slightly decrease (around 10\%) the flexural capacity of RAC beams. Ajdukiewicz and Kliszczewicz [11] used partial or full recycle aggregate. All the beams were fabricated with rectangular sectons measuring $200 \times 300 \mathrm{~mm}$ and $2600 \mathrm{~mm}$ in length with two longitudinal reinforcement ratios of $0.90 \%$ and $1.60 \%$.

They reported that the RAC beams had slightly $3.5 \%$ in average) lower moment capacity and higher deflection compared with the CC beams. Sato et al. [12] tested 37 beams with three different longitudinal reinforcement ratios of $0.59 \%, 1.06 \%$, and $1.65 \%$. They used $100 \%$ recycled aggregate for their mix designs. Results of their study showed that the RAC beams had larger deflection compared to the CC beams. In terms of crack spacing no significant difference observed between the RAC and CC beams. However, the RAC beams had wider crack openings compared to the CC ones. They also reported almost the same ultimate moment for the RAC and CC beams.

Maruyama et al. [49] tested beams with 1\% longitudinal reinforcement ratio and reported that the cracks propagated on the RAC beams were wider and spaced closer compared to those of the CC beams. The RAC beams had larger deflection, but authors reported no significant difference between the flexural capacity of the RAC and CC beams. Fathifazl et al. [15] incorporated the EMV method for their mix designs. They used both $64 \%$ and $74 \%$ RCA as a partial replacement of the coarse aggregate for their RAC mixtures. Their beams were cast with three different longitudinal reinforcement ratios ranging between $0.49 \%$ and $3.31 \%$. Authors reported comparable and even superior flexural behavior for RAC beams at both service and ultimate states. They concluded that the flexural strength provisions of the current codes can be used for RAC beams. Bai \& Sun [36] used 8-10 years old RCA with different replacement levels of $50 \%, 70 \%$, and $100 \%$. They observed similar crack pattern, but deflection and crack width increased with the increment of RCA replacement level. They also concluded that RCA replacement level doesn't significantly affect the cracking ultimate moment of beams. Ignjatovic et al. [20] studied nine full scale beams with $0,50 \%$, and $100 \%$ recycled coarse aggregate and $0.28 \%, 1.46 \%$, and $2.54 \%$ longitudinal reinforcement ratio. They reported no noticeable difference between load-deflection behavior, service load deflection, and ultimate flexural strength of RAC and CC beams. But they observed that the beams with higher range of recycled aggregate showed higher level of concrete destruction at failure.

Kang et al. [25] used beams with longitudinal reinforcement ratio ranged between $0.5 \%$ and $1.8 \%$ with RCA replacement level up to $50 \%$ for both normal and high strength concrete. They observed greater number of cracks and lower cracking moment for RAC beams. They also reported no significant decrease in flexural capacity up to $30 \%$ RCA replacement level. Knaack \& Kurama [32] tested $150 \times 230 \mathrm{~mm}$ cross section and $2000 \mathrm{~mm}$ long beams. They used RCA from late 1920 s foundation and with both $50 \%$ and $100 \%$ replacement level. They reported higher deflection for the RAC beams, but they concluded that the existing analytical models and code provisions can be used for the RAC beams. Arezoumandi et al. [50] replaced 100\% RCA in full scale beams (cross section $300 \times 450 \mathrm{~mm}$ and $3050 \mathrm{~mm}$ long) and reported similar crack pattern and flexural capacity, but lower cracking moment for the RAC beams.

As it can be seen from Table $9(a, b)$, in terms of ultimate moment, when $100 \%$ RCA used as a coarse aggregate, the ACI 318 [5] and EC 2 [7] provisions are conservative for $82 \%$ and $92 \%$ of beams, while only $83 \%$ of the RAC beams showed yielding moment higher than the predicted values. Furthermore, $53 \%$ of the RAC beams showed cracking moment less than the ACI 318 [5] provision; however, it was $39 \%$ when it compared to the EC 2 [7] provision. The ACI 318 [5] and EC 2 [7] ultimate moment provisions are conservative for the beams used less than $30 \%$ RCA as a coarse aggregate, but in terms of cracking and yielding moment $30 \%$ and $60 \%$ of beams showed values less than code predictions, respectively. 
Table 9: Comparison of flexural beam test results with codes.

\begin{tabular}{|c|c|c|c|c|c|c|c|c|c|c|}
\hline Author & Year & Specimen & $\mathbf{M}_{\mathrm{cr}}$ & Test/ACI & Test/EC & My & $\begin{array}{c}\text { Test/ } \\
\text { predicted }\end{array}$ & Mn & Test/ACI & Test/EC \\
\hline \multirow{3}{*}{$\begin{array}{l}\text { Yagishita } \\
\text { et al. [48] }\end{array}$} & \multirow{3}{*}{1994} & BR1 & 12.25 & 1.03 & 0.98 & $\mathrm{NR}^{*}$ & $\mathrm{NR}^{*}$ & 12.25 & 1.03 & 0.98 \\
\hline & & BR2 & 12.65 & 1.06 & 1.02 & $\mathrm{NR}^{*}$ & $\mathrm{NR}^{*}$ & 12.65 & 1.06 & 1.02 \\
\hline & & BR3 & 12.4 & 1.04 & 1 & $\mathrm{NR}^{*}$ & $\mathrm{NR}^{*}$ & 12.4 & 1.04 & 1 \\
\hline \multirow{3}{*}{$\begin{array}{l}\text { Maruyama } \\
\text { et al. [49] }\end{array}$} & \multirow{3}{*}{2004} & CRC30 & 15.3 & 1.12 & 1.14 & 12.5 & 0.98 & 15.3 & 1.12 & 1.14 \\
\hline & & CRC45 & 14.8 & 1.1 & 1.11 & 13.2 & 1.04 & 14.8 & 1.1 & 1.11 \\
\hline & & CRC60 & 15.3 & 1.16 & 1.2 & 12.8 & 1.02 & 15.3 & 1.16 & 1.2 \\
\hline \multirow{15}{*}{$\begin{array}{c}\text { Sato et al. } \\
\text { [12] }\end{array}$} & \multirow{15}{*}{2007} & $\begin{array}{l}\text { CR45-01- } \\
\text { 10WB }\end{array}$ & 7.4 & 1.02 & 1.04 & 7.4 & 1.08 & 7.4 & 1.02 & 1.04 \\
\hline & & $\begin{array}{l}\text { CR45-01- } \\
\text { 10DB }\end{array}$ & 7.1 & 0.98 & 1 & 7.1 & 1.03 & 7.1 & 0.98 & 1 \\
\hline & & $\begin{array}{l}\text { CR60-01- } \\
\text { 10WB }\end{array}$ & 7.7 & 1.06 & 1.08 & 7.7 & 1.12 & 7.7 & 1.06 & 1.08 \\
\hline & & $\begin{array}{l}\text { CR60-01- } \\
\text { 10DB }\end{array}$ & 7.8 & 1.08 & 1.1 & 7.8 & 1.13 & 7.8 & 1.08 & 1.1 \\
\hline & & $\begin{array}{l}\text { CR45-01- } \\
\text { 13WB }\end{array}$ & 12.9 & 0.97 & 1.01 & 12.9 & 1.01 & 12.9 & 0.97 & 1.01 \\
\hline & & $\begin{array}{l}\text { CR45-01- } \\
\text { 13DB }\end{array}$ & 13.2 & 1 & 1.03 & 13.2 & 1.03 & 13.2 & 1 & 1.03 \\
\hline & & $\begin{array}{l}\text { CR60-01- } \\
\text { 13WB }\end{array}$ & 12.5 & 0.93 & 0.98 & 12.5 & 0.97 & 12.5 & 0.93 & 0.98 \\
\hline & & $\begin{array}{l}\text { CR60-01- } \\
\text { 13DB }\end{array}$ & 13.4 & 1 & 1.05 & 13.4 & 1.05 & 13.4 & 1 & 1.05 \\
\hline & & $\begin{array}{c}\text { CR45-01- } \\
16 \mathrm{WB}\end{array}$ & 18.9 & 0.98 & 1.04 & 18.9 & 1 & 18.9 & 0.98 & 1.04 \\
\hline & & $\begin{array}{c}\text { CR45-01- } \\
\text { 16DB }\end{array}$ & 18.9 & 0.99 & 1.05 & 18.9 & 1.01 & 18.9 & 0.99 & 1.05 \\
\hline & & $\begin{array}{c}\text { CR60-01- } \\
\text { 16WB }\end{array}$ & 18.8 & 0.96 & 1.02 & 18.8 & 0.99 & 18.8 & 0.96 & 1.02 \\
\hline & & $\begin{array}{c}\text { CR60-01- } \\
\text { 16DB }\end{array}$ & 19.7 & 1.02 & 1.1 & 19.7 & 1.04 & 19.7 & 1.02 & 1.1 \\
\hline & & $\begin{array}{l}\text { CR30-03- } \\
\text { DB }\end{array}$ & 12.5 & 0.96 & 0.98 & 12.5 & 1.02 & 12.5 & 0.96 & 0.98 \\
\hline & & $\begin{array}{l}\text { CR45-03- } \\
\text { DB }\end{array}$ & 13.2 & 1.03 & 1.04 & 13.2 & 1.09 & 13.2 & 1.03 & 1.04 \\
\hline & & $\begin{array}{l}\text { CR60-03- } \\
\text { DB }\end{array}$ & 12.8 & 1.01 & 1.06 & 12.8 & 1.07 & 12.8 & 1.01 & 1.06 \\
\hline
\end{tabular}

Table 9 (a): Comparison of flexural beam test results with codes.

\begin{tabular}{|c|c|c|c|c|c|c|c|c|c|c|}
\hline Author & Year & Specimen & $\mathbf{M}_{\mathrm{cr}}$ & $\begin{array}{l}\text { Tes t / } \\
\text { ACI }\end{array}$ & Test/EC & My & $\begin{array}{l}\mathrm{T} \text { e } \mathbf{s} \mathrm{t} / \\
\text { predicted }\end{array}$ & Mn & Test/ACI & Test/EC \\
\hline \multirow{8}{*}{$\begin{array}{ll}\text { Ajdukiewicz } & \& \\
\text { Kliszczewicz [11] } & \end{array}$} & \multirow[t]{8}{*}{2007} & ORNl-b1 & 16 & 1.46 & 1.67 & 46.8 & 0.97 & 51.2 & 1.01 & 1.04 \\
\hline & & ORNm-b1 & 12 & 0.86 & 1 & 50.4 & 1.03 & 62.4 & 1.21 & 1.23 \\
\hline & & GRNl-b1 & 12 & 1.02 & 1.14 & 54 & 1.11 & 65.2 & 1.28 & 1.31 \\
\hline & & GRNm-b1 & 16 & 1.11 & 1.29 & 50.8 & 1.04 & 54.4 & 1.05 & 1.07 \\
\hline & & GRNh-b1 & 16 & 0.93 & 1.12 & 52 & 1.05 & 63.2 & 1.21 & 1.24 \\
\hline & & BRNl-b1 & 16 & 1.45 & 1.65 & 52.8 & 1.09 & 60 & 1.18 & 1.21 \\
\hline & & BRNm-b1 & 16 & 1.13 & 1.19 & 49.6 & 1.01 & 57.2 & 1.11 & 1.13 \\
\hline & & BRNh-b1 & 20 & 1.05 & 1.29 & 56 & 1.13 & 66.4 & 1.26 & 1.31 \\
\hline
\end{tabular}




\section{Civil Engineering Research Journal}

\begin{tabular}{|c|c|c|c|c|c|c|c|c|c|c|}
\hline & & ORNl-b2 & 12 & 1.07 & 1.21 & 85.6 & 1.02 & 94.4 & 1.09 & 1.14 \\
\hline & & BRNl-b2 & 18 & 1.62 & 1.84 & 85.6 & 1.02 & 96.4 & 1.12 & 1.18 \\
\hline \multirow[t]{5}{*}{ Bai \& Sun [36] } & 2010 & RAC-2 & 10 & 1.1 & 1.22 & 28 & 1.06 & 34 & 1.22 & 1.24 \\
\hline & & RAC-3 & 11.2 & 1.21 & 1.34 & 28 & 1.06 & 35.5 & 1.27 & 1.3 \\
\hline & & RAC-4 & 10 & 1.09 & 1.2 & 26.5 & 1 & 34 & 1.22 & 1.24 \\
\hline & & RAC-5 & 10.6 & 1.15 & 1.27 & 32.5 & 0.95 & 40.4 & 1.12 & 1.16 \\
\hline & & RAC-6 & 10.6 & 1.15 & 1.27 & 40 & 1.01 & 48.4 & 1.17 & 1.21 \\
\hline \multirow[t]{8}{*}{ Fathifazl et al. [15] } & 2009 & EM-Min & 13 & 0.85 & 0.96 & 38.4 & 1.21 & 46 & 1.39 & 1.42 \\
\hline & & $\mathrm{EM}-\mathrm{Av}$ & 13.8 & 0.9 & 1.02 & 123.4 & 1.01 & 149.2 & 1.2 & 1.3 \\
\hline & & EM-Max & 21.1 & 1.11 & 1.25 & 210.6 & 0.97 & 221.9 & 1.1 & 1.22 \\
\hline & & EM-СMP & 24.7 & 1.61 & 1.82 & 193 & 0.9 & 246.1 & 1.24 & 1.38 \\
\hline & & EV-Min & 16.2 & 0.97 & 1.07 & 39.9 & 1.25 & 46.7 & 1.4 & 1.44 \\
\hline & & $\mathrm{EV}-\mathrm{Av}$ & 15.2 & 0.91 & 1 & 136.6 & 1.11 & 150.2 & 1.19 & 1.25 \\
\hline & & EV-Max & 13.4 & 0.65 & 0.71 & 207.5 & 0.95 & 225.2 & 1.07 & 1.17 \\
\hline & & EV-CMP & 29.1 & 1.74 & 1.92 & 220.2 & 1.02 & 245.7 & 1.19 & 1.3 \\
\hline \multirow{4}{*}{$\begin{array}{l}\text { Arezoumandi et al. } \\
{[50]}\end{array}$} & 2015 & F-6-1 & 34.6 & 0.94 & 1.1 & 119.3 & 0.99 & 149.6 & 1.21 & 1.25 \\
\hline & & F-6-2 & 35.9 & 0.96 & 1.12 & 125.4 & 1.04 & 154.9 & 1.25 & 1.29 \\
\hline & & F-7-1 & 42 & 1.14 & 1.33 & 147.4 & 1 & 172.6 & 1.14 & 1.19 \\
\hline & & F-7-2 & 42.7 & 1.14 & 1.33 & 148.2 & 1.01 & 169.3 & 1.12 & 1.16 \\
\hline
\end{tabular}

Table 9 (b): Comparison of flexural beam test results with codes.

\begin{tabular}{|c|c|c|c|c|c|c|c|c|c|c|}
\hline Author & Year & Specimen & Mcr & Test/ACI & Test/EC & My & $\begin{array}{c}\text { Test/ } \\
\text { predicted }\end{array}$ & $\mathrm{Mn}$ & Test/ACI & Test/EC \\
\hline \multirow[t]{20}{*}{$\begin{array}{c}\text { Kang et al. } \\
\text { [25] }\end{array}$} & 2014 & H15-0.5 & 5.8 & 0.73 & 0.86 & 13 & 1.02 & 18.2 & 1.35 & 1.4 \\
\hline & & H15-1.0 & 6.6 & 0.84 & 0.98 & 21.7 & 0.96 & 29.3 & 1.23 & 1.25 \\
\hline & & H15-1.5 & 7.9 & 1.01 & 1.18 & 30.2 & 0.94 & 38.6 & 1.13 & 1.15 \\
\hline & & H15-1.8 & 9.1 & 1.16 & 1.35 & 45.2 & 0.96 & 58.1 & 1.17 & 1.23 \\
\hline & & Н30-0.5 & 5.3 & 0.74 & 0.8 & 11.2 & 0.88 & 16.9 & 1.26 & 1.3 \\
\hline & & Н30-1.0 & 6.2 & 0.87 & 0.95 & 21.9 & 0.97 & 28.8 & 1.21 & 1.24 \\
\hline & & Н30-1.5 & 7 & 0.99 & 1.07 & 31.1 & 0.96 & 38.4 & 1.13 & 1.17 \\
\hline & & Н30-1.8 & 7.9 & 1.11 & 1.21 & 46.9 & 1 & 49 & 1 & 1.05 \\
\hline & & N15-0.5 & 6.6 & 1.14 & 1.31 & 10.9 & 0.86 & 14.8 & 1.12 & 1.14 \\
\hline & & N15-1.0 & 8 & 1.37 & 1.58 & 22.6 & 1.01 & 27.2 & 1.17 & 1.22 \\
\hline & & N15-1.5 & 8.7 & 1.5 & 1.73 & 30.6 & 0.96 & 35.8 & 1.09 & 1.14 \\
\hline & & N15-1.8 & 12.4 & 2.13 & 2.46 & 47.3 & 1.02 & 51.6 & 1.11 & 1.2 \\
\hline & & N30-0.5 & 5.7 & 1 & 1.16 & 12.5 & 0.99 & 14.7 & 1.11 & 1.13 \\
\hline & & N30-1.0 & 8 & 1.39 & 1.62 & 23.5 & 1.05 & 26.3 & 1.14 & 1.18 \\
\hline & & N30-1.5 & 8.4 & 1.46 & 1.7 & 32.4 & 1.02 & 35.3 & 1.08 & 1.15 \\
\hline & & N30-1.8 & 12.4 & 2.16 & 2.51 & 44 & 0.95 & 50.2 & 1.09 & 1.2 \\
\hline & & N50-0.5 & 6.6 & 1.21 & 1.42 & 11.1 & 0.88 & 13.6 & 1.03 & 1.04 \\
\hline & & N50-1.0 & 8 & 1.45 & 1.71 & 22.6 & 1.02 & 24.4 & 1.06 & 1.09 \\
\hline & & N50-1.5 & 8.8 & 1.61 & 1.9 & 32.3 & 1.02 & 32.8 & 1.01 & 1.07 \\
\hline & & N50-1.8 & 11 & 2.01 & 2.37 & 48.8 & 1.05 & 50.5 & 1.11 & 1.18 \\
\hline
\end{tabular}




\section{Civil Engineering Research Journal}

Figure 4 presents the normalized flexural strength versus normalized longitudinal reinforcement ratio for the RAC beams as well as the wealth of flexural test data available in the literature for CC [51]. Figure 4 seems to indicate that the RAC and CC test results fall within the central portion of the data. Furthermore, statistical analysis (regression analysis) of the data indicates that the RAC and CC test results fall within a 95\% confidence interval of a nonlinear regression curve fit of the database. This result indicates that the RAC flexural test values are very consistent with the wealth of flexural test data available in the literature.

\section{Shear Strength}

Mukai \& Kikuchi [41] replaced both $15 \%$ and $30 \%$ RCA instead of virgin aggregate and reported slightly inferior shear strength for the RAC compared with the CC beams. Han et al. [52] tested beams with different shear span to depth (a/d=1.5 to 4.0) and both washed and unwashed RCA and observed no significant difference in failure mode and shear capacity between the RAC and CC beams; however, the ACI 318 [5] shear provision was unconservative for beams with a/d greater than 3.0. Yagashita et al. [48] used low, medium, and high grade RCA with 100\% replacement level. They reported $8 \%$ lower shear stress at failure for the RAC beams (with high grade RCA) compared to the CC. Sogo et al. [53] used RCA (10 to 40-year-old concrete foundation) for casting the beams and reported $20 \%$ lower shear strength for the RAC beams compared to the CC beams. Gonza'lez-Fonteboa et al. [54] tested beams with 50\% RCA replacement and observed no significant difference on shear capacity between the RAC and CC beams. Etxeberria \& Va'zquez [55] used different percentages of RCA (25\%, 50\%, and 100\%) for casting specimens and concluded that a substitution of less than $25 \%$ RCA, scarcely affects the shear capacity of RC beams. Ji et al. [56] tested beams with a cross section of $170 \times 270 \mathrm{~mm}$ and a shear span-to-depth of 2 and 100\% RCA replacement. Results of their study showed that existing codes can be used to predict shear strength of the RAC beams. Choi et al. [16] tested beams with different span-to-depth ratios (1.50, 2.50 and 3.25) and different RCA replacement ratios (30\%, 50\%, and 100\%). They reported the lower shear strength for the RAC beams. Fathifazl et al. [57] used the EMV method with both $63.5 \%$ and $74.3 \%$ recycled aggregate replacement as a coarse aggregate for their mix designs. They tested beams with four different shear spanto-depth ratios ranging between 1.5 and 4. They reported superior shear strength for the RAC beams. They also concluded that current code provisions for shear conservatively predicted the capacities of the RAC beams. Schubert et al. [30] tested slabs with $100 \%$ RCA replacement and concluded that existing codes provision can be used to calculate shear strength of the RAC slabs. Xiao et al. [58] tested push-off specimens with different percentages of recycled coarse aggregate replacement. They observed similar shear stress-slip curves and crack propagation path between the RAC and CC specimens. They also reported that more than 30\% RCA replacement decrease the ultimate shear capacity. Arezoumandi et al. [59] replaced 100\% RCA in full scale beams with different longitudinal reinforcement ratios and reported $12 \%$ lower shear strength for the RAC beams compared to the CC beams. Knaack \& Kurama [32] replaced both $50 \%$ and $100 \%$ of RCA (from 90 years old building) to cast beams with cross section of $150 \mathrm{~mm} \times 230 \mathrm{~mm}$. Their results showed small reduction on shear strength of the RAC beams, but still existing codes can be used to predict shear strength of the RAC beams.

Table 10: Summary of shear beam test results

\begin{tabular}{|c|c|c|c|c|c|c|c|c|c|c|c|c|}
\hline Author & year & RCA (\%) & b (mm) & $\begin{array}{c}\mathrm{d} \\
(\mathrm{mm})\end{array}$ & $h(\mathrm{~mm})$ & $a / d$ & $\underset{(\mathrm{mm})}{\mathrm{d}_{\max }}$ & $\rho(\%)$ & fc Mpa & V kN & $\begin{array}{l}\text { Test/ } \\
\text { ACI }\end{array}$ & $\begin{array}{c}\text { Test/ } \\
\text { EC }\end{array}$ \\
\hline & \multirow{5}{*}{2001} & \multirow{5}{*}{100} & 170 & 270 & 300 & 1.5 & 25 & 1.1 & 39.6 & 83.5 & 1.7 & 2.32 \\
\hline & & & 170 & 270 & 300 & 2 & 25 & 1.1 & 30.6 & 65.2 & 1.51 & 1.97 \\
\hline & & & 170 & 270 & 300 & 2 & 25 & 1.1 & 32.6 & 60.6 & 1.36 & 1.79 \\
\hline & & & 170 & 270 & 300 & 3 & 25 & 1.1 & 31.2 & 42.7 & 0.98 & 1.28 \\
\hline & & & 170 & 270 & 300 & 4 & 25 & 1.1 & 31.9 & 31.7 & 0.72 & 0.94 \\
\hline $\begin{array}{c}\text { Belén \& Fernando } \\
{[3]}\end{array}$ & 2007 & 50 & 200 & 303 & 350 & 3.3 & 25 & 3 & 39.7 & 90.6 & 1.4 & 1.6 \\
\hline \multirow{3}{*}{$\begin{array}{c}\text { Etxeberria \& } \\
\text { Va'zquez [10] }\end{array}$} & \multirow{3}{*}{2007} & 25 & 200 & 303 & 350 & 3.3 & 25 & 2.9 & 42.4 & 104 & 1.55 & 1.8 \\
\hline & & 50 & 200 & 303 & 350 & 3.3 & 25 & 2.9 & 41.3 & 89 & 1.34 & 1.55 \\
\hline & & 100 & 200 & 303 & 350 & 3.3 & 25 & 2.9 & 39.8 & 84 & 1.29 & 1.48 \\
\hline Ji et al. [56] & 2008 & 100 & 170 & 270 & 300 & 2.2 & $\mathrm{NR}^{*}$ & 1.1 & 39.7 & 60 & 1.22 & 1.66 \\
\hline \multirow{5}{*}{ Choi et al. [16] } & \multirow{5}{*}{2010} & \multirow{3}{*}{30} & 200 & 360 & 400 & 1.5 & 25 & 1.6 & 24.6 & 161.7 & 2.66 & 3.15 \\
\hline & & & 200 & 360 & 400 & 2.5 & 25 & 1.6 & 24.6 & 81.4 & 1.34 & 1.59 \\
\hline & & & 200 & 360 & 400 & 3.25 & 25 & 1.6 & 24.6 & 80.9 & 1.33 & 1.58 \\
\hline & & \multirow{2}{*}{50} & 200 & 360 & 400 & 1.5 & 25 & 1.6 & 24.2 & 152.9 & 2.54 & 3 \\
\hline & & & 200 & 360 & 400 & 2.5 & 25 & 1.6 & 24.2 & 87.9 & 1.46 & 1.72 \\
\hline
\end{tabular}




\section{Civil Engineering Research Journal}

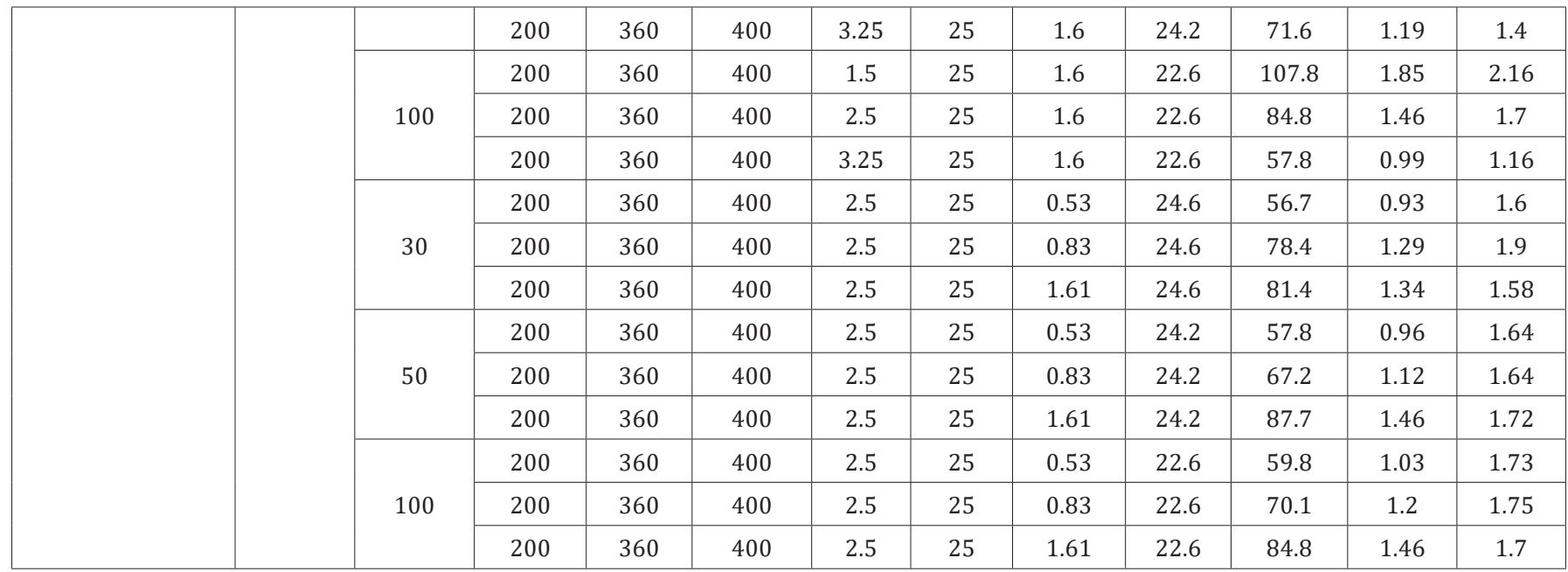

Table 10 (a): Summary of shear beam test results

\begin{tabular}{|c|c|c|c|c|c|c|c|c|c|c|c|c|}
\hline Author & year & $\begin{array}{l}\text { RCA } \\
\text { (\%) }\end{array}$ & $\begin{array}{c}\mathbf{b} \\
(\mathrm{mm})\end{array}$ & $\begin{array}{c}d \\
(\mathrm{~mm})\end{array}$ & $\begin{array}{c}\mathbf{h} \\
(\mathrm{mm})\end{array}$ & $a / d$ & $\begin{array}{l}\text { dmax } \\
(\mathrm{mm})\end{array}$ & $\rho(\%)$ & $\begin{array}{c}\text { fc } \\
\text { Mpa }\end{array}$ & V kN & $\begin{array}{c}\text { Test/ } \\
\text { ACI }\end{array}$ & Test/EC \\
\hline \multirow{14}{*}{ Fathifazl et al. [57] } & \multirow{14}{*}{2011} & 63.5 & 200 & 300 & 375 & 1.5 & 19 & 1 & 41.6 & 186.7 & 2.84 & 4.12 \\
\hline & & & 200 & 300 & 375 & 2 & 19 & 1.5 & 41.6 & 169.5 & 2.58 & 3.27 \\
\hline & & & 200 & 309 & 375 & 2.7 & 19 & 1.62 & 41.6 & 103.9 & 1.53 & 1.91 \\
\hline & & & 200 & 305 & 375 & 4 & 19 & 2.46 & 41.6 & 83.2 & 1.24 & 1.44 \\
\hline & & 74.3 & 200 & 300 & 375 & 1.5 & 19 & 1 & 49.1 & 195.3 & 2.73 & 4.08 \\
\hline & & & 200 & 300 & 375 & 2 & 19 & 1.5 & 49.1 & 179 & 2.5 & 3.27 \\
\hline & & & 200 & 305 & 375 & 4 & 19 & 2.46 & 49.1 & 105.6 & 1.45 & 1.73 \\
\hline & & 63.5 & 200 & 201 & 250 & 2.7 & 19 & 2 & 41.6 & 89.3 & 2.03 & 2.12 \\
\hline & & & 200 & 309 & 375 & 2.6 & 19 & 1.6 & 41.6 & 103.9 & 1.53 & 1.92 \\
\hline & & & 200 & 381 & 450 & 2.7 & 19 & 1.8 & 41.6 & 99.5 & 1.19 & 1.5 \\
\hline & & & 200 & 476 & 550 & 2.7 & 19 & 1.7 & 41.6 & 104.6 & 1 & 1.34 \\
\hline & & 74.3 & 200 & 201 & 250 & 2.7 & 19 & 2 & 49.1 & 122.6 & 2.56 & 2.76 \\
\hline & & & 200 & 381 & 450 & 2.7 & 19 & 1.8 & 49.1 & 111.7 & 1.23 & 1.59 \\
\hline & & & 200 & 476 & 550 & 2.7 & 19 & 1.7 & 49.1 & 119.6 & 1.05 & 1.45 \\
\hline \multirow{8}{*}{ Knaack \& Kurama [32] } & \multirow{8}{*}{2014} & 50 & 150 & 200 & 230 & 3.8 & 19 & 1.3 & 41.8 & 44 & 1.33 & 1.61 \\
\hline & & & 150 & 200 & 230 & 3.8 & 19 & 1.3 & 41.8 & 39.1 & 1.19 & 1.43 \\
\hline & & & 150 & 200 & 230 & 3.8 & 19 & 1.3 & 37.4 & 43.7 & 1.4 & 1.66 \\
\hline & & & 150 & 200 & 230 & 3.8 & 19 & 1.3 & 37.4 & 41.2 & 1.32 & 1.57 \\
\hline & & 100 & 150 & 200 & 230 & 3.8 & 19 & 1.3 & 39.1 & 36.4 & 1.14 & 1.36 \\
\hline & & & 150 & 200 & 230 & 3.8 & 19 & 1.3 & 39.1 & 38 & 1.19 & 1.42 \\
\hline & & & 150 & 200 & 230 & 3.8 & 19 & 1.3 & 39.2 & 39.9 & 1.25 & 1.49 \\
\hline & & & 150 & 200 & 230 & 3.8 & 19 & 1.3 & 39.2 & 36.1 & 1.13 & 1.35 \\
\hline \multirow{6}{*}{ Arezoumandi et al. [59] } & \multirow{6}{*}{2014} & 100 & 305 & 400 & 460 & 3.1 & 25 & 1.27 & 30 & 114.8 & 1.01 & 1.37 \\
\hline & & & 305 & 375 & 460 & 3.25 & 25 & 1.27 & 30 & 143.2 & 1.34 & 1.79 \\
\hline & & & 305 & 375 & 460 & 3.25 & 25 & 2.03 & 30 & 131.4 & 1.23 & 1.41 \\
\hline & & & 305 & 400 & 460 & 3.1 & 25 & 2.03 & 34.1 & 113 & 0.93 & 1.11 \\
\hline & & & 305 & 375 & 460 & 3.25 & 25 & 2.71 & 34.1 & 124.1 & 1.09 & 1.28 \\
\hline & & & 305 & 375 & 460 & 3.25 & 25 & 2.71 & 34.1 & 140.3 & 1.24 & 1.45 \\
\hline
\end{tabular}


Table 10 summarizes details of shear beams test including width, b, height, h, depth, d, shear span to depth, a/d, maximum aggregate size, dmax, longitudinal reinforcement ratio, $\rho$, compressive strength, $\mathrm{f}^{\prime} \mathrm{c}$, and shear at failure, V. Furthermore, test results compared with shear provisions of both ACI 318 [5] and EC 2 [7]. Table 10 (a) shows that both ACI 318 [5] and EC 2 [7] shear provisions are conservative for more than $90 \%$ of data, even though for the majority of tests the RAC beams showed lower shear strength compared to the CC beams.

The four key parameters that affect concrete contribution to shear strength include depth of member or size effect (d), shear span to depth ratio $(a / d)$, compressive strength of concrete $\left(f^{\prime} c\right)$, and longitudinal reinforcement ratio $(\rho)$. To evaluate the effect of the aforementioned parameters on shear strength of the beams, the results of RAC beams are compared with the wealth of shear test data available in the literature for CC [60].
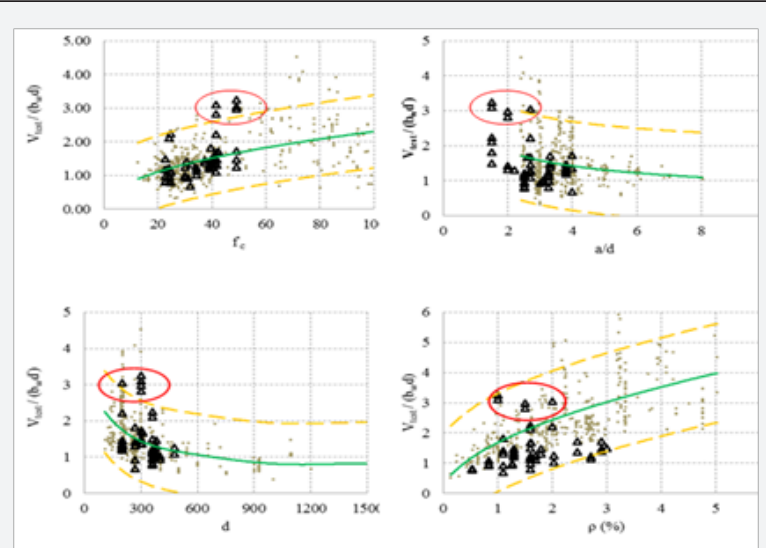

Figure 5: Comparison of database for shear strength of RAC and CC [60].

Figure 5 presents the shear stress versus $f^{\prime} c, \rho, d$ and $a / d$, respectively. Given the significant scatter of the database of previous shear test results, it is somewhat difficult to draw definitive conclusions on the current test values. Nonetheless, visually, Figure 5 seems to indicate that the RAC test results fall within the lower portion of the data -except results of Fathifazl et al. [56] - and follow the same general trend of the database. Furthermore, statistical analysis (using regression analysis to draw the best fit and $95 \%$ confidence intervals) of the data indicates that the test results fall within a 95\% confidence interval of a nonlinear regression curve fit of the database. This result indicates that the RAC beams shear strength is lower than the shear strength of the CC.

\section{Conclusion}

The following conclusions can be drawn from literature review of mechanical properties and structural behavior of RAC specimens:

a. The existing codes provisions are unconservative for splitting tensile strength of RAC around $10 \%$. b. The ACI 318 [4] and EC 2 [7] provisions overestimate flexural strength of RAC about $24 \%$ and $65 \%$, respectively.

c. Moduli of elasticity of RAC are around $10 \%$ to $20 \%$ lower than the predicted values of the ACI 318 [4] and EC 2 [7] codes provisions.

d. Moduli of elasticity of RAC are around $10 \%$ to $20 \%$ lower than the predicted values of the ACI 318 [4] and EC 2 [7] codes provisions.

e. The CEB-FIP [5] and Japan's code fracture energy provisions are unconservative for $90 \%$ and $20 \%$ of RAC, respectively.

f. Pull-out specimens with different RCA replacement levels showed almost identical load-slip behavior with no significant difference in bond strength between RAC and CC specimens.

g. Both splice beam and end beam results showed up to $20 \%$ reduction in bond strength of RAC with $100 \%$ RCA replacement.

h. In terms of flexural strength, RAC beams showed wider cracks, lower cracking moment, larger deflection, but similar ultimate moment with CC.

i. The RAC beams test results fall within a central portion of $95 \%$ confidence interval of a nonlinear regression curve fit of a CC flexural test database.

j. Similar load-deflection for shear behavior observed between RAC and CC beams, but the majority of previous studies reported lower shear strength for RAC beams (up to $20 \%$ when $100 \%$ RCA used instead of virgin aggregate) compared to CC.

k. The RAC beams test results fall within a lower portion of $95 \%$ confidence interval of a nonlinear regression curve fit of a CC shear test database.

l. Using the EMV method avoids inferior mechanical and structural performance of RAC.

\section{References}

1. World Business Council for Sustainable Development WBCSD Report (2012) Recycling concrete.

2. Buck AD (1977) Recycled Concrete as a Source of Aggregate. Journal of the American Concrete Institute 74(5): 212-219.

3. Bellen G, Fernando M (2007) Shear strength of recycled concrete beams. Construction and Building Materials 21(4): 887-893.

4. American Concrete Institute ACI Committee (2011) Building code requirements for structural concrete ACI 318-11 and commentary 318R-11. Farmington Hills, MI, USA: American Concrete Institute.

5. CEB-FIP (2012) Model Code 2010 - Final draft, Volume 1. Ernst \& Sohn, Berlin, Germany, p. 350.

6. European Committee for Standardization (2005) Eurocode No 2 Design of concrete structures. Part 1: General Rules and Rules for Buildings. 


\section{Civil Engineering Research Journal}

7. Japan Society of Civil Engineers (2007) Standard Specification for Concrete Structure. Japanese Society of Civil Engineering No. 15, Tokyo, Japan.

8. Sagoe-Crentsil KK, Brown T, Taylor AH (2001) Performance of concrete made with commercially produced coarse recycled concrete aggregate. Cement and Concrete Research 31(5): 707-712.

9. Ajdukiewicz A, Kliszczewicz A (2002) Influence of recycled aggregates on mechanical properties of HS/HPC. Cement and concrete composites 24(2): 269-279.

10. Etxeberria M, Mari AR, Vazquez E (2007) Recycled aggregate concrete as structural material. Materials and Structures 40(5): 529-541.

11. Ajdukiewicz AB, Kliszczewicz AT (2007) Comparative tests of beams and columns made of recycled aggregate concrete and natura aggregate concrete. Journal of Advanced Concrete Technology 5(2): 259-273.

12. Sato R, Maruyama I, Sogabe T, Sogo M (2007) Flexural behavior of reinforced recycled concrete beams. Journal of Advanced Concrete Technology 5(1): 43-61.

13. Yang KH, Chung HS, Ashour AF (2008) Influence of type and replacement level of recycled aggregates on concrete properties. ACI Materials Journal 105(3): 289-296.

14. Tabsh SW, Abdelfatah AS (2009) Influence of recycled concrete aggregates on strength properties of concrete. Construction and Building Materials 23(2): 1163-1167.

15. Fathifazl G, Razaqpur AG, Isgor OB, Abbas A, Fournier B, et al. (2009) Flexural performance of steel-reinforced recycled concrete beams. ACI Structural Journal 106(06): 858-867.

16. Choi HB, Yi CK, Cho HH, Kang KI (2010) Experimental study on the shear strength of recycled aggregate concrete beams. Magazine of Concrete Research 62(2): 103-114.

17. Butler L, West JS, Tighe SL (2011) The effect of recycled concrete aggregate properties on the bond strength between RCA concrete and steel reinforcement. Cement and Concrete Research 41(10): 10371049.

18. Kou SC, Poon CS, Etxeberria M (2011) Influence of recycled aggregates on long term mechanical properties and pore size distribution of concrete. Cement and concrete composites 33(2): 286-291.

19. Abd Elhakam A, Mohamed AE, Awad E (2012) Influence of self-healing, mixing method and adding silica fume on mechanical properties of recycled aggregates concrete. Construction and Building Materials 35: 421-427.

20. Ignjatović IS, Marinković SB, Mišković ZM, Savić AR (2012) Flexural behavior of reinforced recycled aggregate concrete beams under short-term loading. Materials and structures 46(6): 1045-1059.

21. Butler L, West JS, Tighe SL (2013) Effect of recycled concrete coarse aggregate from multiple sources on the hardened properties of concrete with equivalent compressive strength. Construction and Building Materials 47: 1292-1301.

22. Kim Sw, Yum HD (2013) Influence of recycled coarse aggregates on the bond behavior of deformed bars in concrete. Engineering Structures 48 : 133-143.

23. Kou SC, Poon CS (2013) Long-term mechanical and durability properties of recycled aggregate concrete prepared with the incorporation of fly ash. Cement and Concrete Composite 37(1): 12-19.

24. Arezoumandi M, Jonathan Drury J, Volz, JS (2014) Effect of Recycled Concrete Aggregate Replacement Level on the Fracture Behavior of Concrete. Journal of Frontiers in Construction Engineering 3(1): 1-8.
25. Kang THK, Kim W, Kwak Y, Hong S (2014) Flexural Testing of Reinforced Concrete Beams with Recycled Concrete Aggregates. ACI Structural Journal 111(3): 607-616

26. Folino P, Xargay, H (2014) Recycled aggregate concrete-Mechanical behavior under uniaxial and triaxial compression. Construction and Building Materials 56: 21-31.

27. Butler LJ, West JS, Tighe SL (2015) Bond of Reinforcement in Concrete Incorporating Recycled Concrete Aggregates. Journal of Structural Engineering 141(3): 2015.

28. Steele AR (2014) Bond performance of recycled aggregate concrete. Master thesis, Rolla, MO, USA.

29. Katz A (2003) Properties of concrete made with recycled aggregate from partially hydrated old concrete. Cement and concrete research 33(5): 703-711.

30. Schubert S, Hoffmann C, Leemann A, Moser k, Motavalli M (2012) Recycled aggregate concrete: Experimental shear resistance of slabs without shear reinforcement. Engineering Structures 41: 490-497.

31. Andreu G, Miren E (2014) Experimental analysis of properties of high performance recycled aggregate concrete. Construction and Building Materials 52: 227-235.

32. Knaack AM, Kurama YC (2014) Behavior of Reinforced Concrete Beams with Recycled Concrete Coarse Aggregates. Journal of Structural Engineering 141(30): 2015

33. Casuccio M, Torrijos MC, Giaccio, G, Zerbino R (2008) Failure mechanism of recycled aggregate concrete. Construction and Building Materials 22 (7): 1500-1506.

34. Berndt ML (2009) Properties of sustainable concrete containing fly ash, slag and recycled concrete aggregate. Construction and Building Materials 23(7): 2606-2613.

35. Debieb F, Courard L, Kenai S, Degeimbre R (2010) Mechanical and durability properties of concrete using contaminated recycled aggregates. Cement and Concrete composites 32(6): 421-426.

36. Bai WH, Sun BX (2010) Experimental Study on Flexural Behavior of Recycled Coarse Aggregate Concrete Beam. Applied Mechanics and Materials 29: 543-548.

37. Zega, C J, Di Maio AA (2011) Recycled Concretes Made with Waste Ready-Mix Concrete as Coarse Aggregate. Journal of Materials in Civil Engineering 23(3): 281-286.

38. Kou SC, Poon CS, Agrela F (2011) Comparisons of natural and recycled aggregate concretes prepared with the addition of different mineral admixtures. Cement and Concrete Composites 33(8): 788-795.

39. Cui H, Shi X, Memon S, Xing F, Tang W, et al. (2014) Experimental Study on the Influence of Water Absorption of Recycled Coarse Aggregates on Properties of the Resulting Concretes. J Mater Civ Eng 27(4): 1-9.

40. American Concrete Institute ACI Committee (2003) Bond and Development of Straight Reinforcing Bars in Tension (ACI 408R-03). Farmington Hills, MI: American Concrete Institute.

41. Mukai T, Kikuchi M (1988) Properties of reinforced concrete beams containing recycled aggregate. Demolition and reuse of concrete and masonry 2: 670-679.

42. RILEM 7-II-128 (1994) RC6: Bond Test for Reinforcing Steel. 1. PullOut Test. RILEM technical recommendations for the testing and use of construction materials, E \& FN Spon, UK, pp.102-105.

43. Xiao J, Falkner H (2007) Bond behaviour between recycled aggregate concrete and steel rebars. Construction and Building Materials 21(2): 395-401. 


\section{Civil Engineering Research Journal}

44. Breccolotti M, Materazzi AL (2013) Structural reliability of bonding between steel rebars and recycled aggregate concrete. Construction and Building Materials 47: 927-934.

45. CSA CAN3-A23.3-2004 (2004) Design of Concrete Standards for Buildings. Rexdale, Ontario, Canada.

46. Prince MJR, Singh B (2014) Bond behaviour between recycled aggregate concrete and deformed steel bars. Materials and Structures, 47(3): 503-516.

47. Fathifazl G (2008) Structural performance of steel reinforced recycled concrete members. Doctoral dissertation, University of Ottawa, Canada, p. 1-504

48. Yagishita F, Sano M, Yamada M (1994) Behavior of Reinforced Concrete Beams containing Recycled Coarse Aggregate. Journal of Structural Engineering 141(3): 331-342.

49. Maruyama I, Sogo M, Sogabe T, Sato R, Kawai K (2004) Flexural properties of reinforced recycled concrete beams. Internacional RILEM conference on the use of recycled materials in buildings and structures, Hiroshima University, Japan, p.1-10.

50. Arezoumandi M, Smith A, Volz JS, Khayat KH (2014) An experimental study on flexural strength of reinforced concrete beams with $100 \%$ recycled concrete aggregate. Engineering Structures 88: 154-162.

51. Kenneth L, Dionisio B (1997) Reinforced Concrete Design. ( ${ }^{\text {rd }}$ edn), McGraw Hill International edition, India.

52. Han BC, Yun HD, Chung SY (2001) Shear capacity of reinforced concrete beams made with recycled-aggregate. ACI Special publication 200: 503-516.
53. Sogo M, Sogabe T, Maruyma I, Sato R, Kawai K (2004) Shear behavior of reinforced recycled concrete beams. Proceedings of the International RILEM Conference on the Use of Recycled Materials in Building and Structures, Hiroshima University, Japan, p. 610-618.

54. Gonzalez-Fonteboa B, Martinez-Abella F (2007) Shear strength of recycled concrete beams. Construction and Building materials 21(4): 887-893.

55. Etxeberria M, Vázquez E, Marí A, Barry M (2007) Influence of amount of recycled coarse aggregates and production process on properties of recycled aggregate concrete. Cement and concrete research 37: 735742.

56. Ji SK, Lee WS, DoYun H (2008) Shear strength of reinforced concrete beams with recycled aggregates. In: Walraven \& Stoelhorst (eds), Tailor Made Concrete Structures, Taylor \& Francis Group, London, p. 247.

57. Fathifazl G, Razaqpur AG, Burkan IO, Abbas A, Fournier B, et al. (2011) Shear capacity evaluation of steel reinforced recycled concrete (RRC) beams. Engineering Structures 33(3): 1025-1033.

58. Xiao J, Xie H, Yang Z (2012) Shear transfer across a crack in recycled aggregate concrete. Cement and Concrete Research 42(5): 700-709.

59. Arezoumandi M, Smith A, Volz JS, Khayat KH (2014) An experimental study on shear strength of reinforced concrete beams with $100 \%$ recycled concrete aggregate. Construction and Building Materials 53: 612-620.

60. Reineck KH, Kuchma DA, Kim KS, and Marx S (2003) Shear Database for Reinforced Concrete Members without Shear Reinforcement. ACI Structural Journal 100 (2): 240-249.

\section{Your next submission with Juniper Publishers will reach you the below assets}

- Quality Editorial service

- Swift Peer Review

- Reprints availability

- E-prints Service

- Manuscript Podcast for convenient understanding

- Global attainment for your research

- Manuscript accessibility in different formats

( Pdf, E-pub, Full Text, Audio)

- Unceasing customer service

Track the below URL for one-step submission https://juniperpublishers.com/online-submission.php 